\title{
Person perception informs understanding of cognition during visual search
}

\author{
Allison A. Brennan • Marcus R. Watson • \\ Alan Kingstone • James T. Enns
}

Published online: 28 May 2011

(C) Psychonomic Society, Inc. 2011

\begin{abstract}
Does person perception-the impressions we form from watching others - hold clues to the mental states of people engaged in cognitive tasks? We investigated this with a two-phase method: In Phase 1, participants searched on a computer screen (Experiment 1) or in an office (Experiment 2); in Phase 2, other participants rated the searchers' video-recorded behavior. The results showed that blind raters are sensitive to individual differences in search proficiency and search strategy, as well as to environmental factors affecting search difficulty. Also, different behaviors were linked to search success in each setting: Eye movement frequency predicted successful search on a computer screen; head movement frequency predicted search success in an office. In both settings, an active search strategy and positive emotional expressions were linked to search success. These data indicate that person perception informs cognition beyond the scope of performance measures, offering the potential for new measurements of cognition that are both rich and unobtrusive.
\end{abstract}

Keywords Visual search - Eye movements and visual attention $\cdot$ Attention

"You can observe a lot by just watching."

-Widely attributed to Yogi Berra

A typical experiment in cognitive psychology involves the presentation of a stimulus in a controlled laboratory setting, systematic variation of the conditions under which the stimulus is presented, and measurement of the partic-

A. A. Brennan $(\bowtie) \cdot$ M. R. Watson $\cdot$ A. Kingstone $\cdot$ J. T. Enns Department of Psychology, University of British Columbia, Vancouver, British Columbia, Canada V6T 1Z4

e-mail: abrennan@psych.ubc.ca ipant's response with a combination of keypresses, brief vocal responses, eye movements, and limb actions. Cognitive researchers almost never look directly at participants while they perform in an experiment, leaving it an open question whether they are missing key features of visible behavior that are relevant to the mental processes under investigation. The purpose of the present study is to ask whether researchers can enhance their understanding of cognition by adding measures of person perception to their standard toolbox of performance measurements.

Our motivation is both practical and theoretical. On the practical side, most personal computers today come equipped with a built-in webcam aimed directly at the user. We can think of no reason why this resource should lie dormant without consideration of its research potential. Theoretically, many studies in social psychology over the past decade have demonstrated the surprising reliability and validity of thin-slicing, referring to the ability of persons to make rapid evaluations of the personality, disposition, and intent of others from very small samples of their behavior (Ambady, Bernieri, \& Richeson, 2000; Ambady, Hallahan, \& Rosenthal, 1995; Borkenau \& Liebler, 1995; Borkenau, Mauer, Riemann, Spinath, \& Angleitner, 2004; Carney, Colvin, \& Hall, 2007; Gladwell, 2007; Naumann, Vazire, Rentfrow, \& Gosling, 2009; Rule, Macrae, \& Ambady, 2009; Weisbuch \& Ambady, 2011). Why should cognitive researchers not also consider this potential signal?

A second theoretical motivation comes from the growing interest in emotional, social, and motivational influences on cognitive performance - what are often referred to as "warm" factors - over and above the "cold" factors of stimulus timing, strength, and probability. For example, following the induction of various mood states, participants in a happy mood make more frequent saccades and fixate longer on positive emotional peripheral stimuli than do 
control participants (Wadlinger \& Isaacowitz, 2006); happy participants are more likely than sad participants to selectively process patterns at a configurational level in global-local tasks (Fredrickson, 2003; Gasper \& Clore, 2002); happy participants are less likely than anxious participants to experience an attentional blink in a rapid serial visual presentation (Jefferies, Smilek, Eich, \& Enns, 2008); and anxious participants are more likely to have a narrow spatial focus of attention than are control participants (Derryberry \& Reed, 1998; Derryberry \& Tucker, 1994). Importantly, the direction of the emotion-performance link can run the other way as well. Flow theory is a way to account for the observation that skilled, fluid performances in many domains of life are associated with positive emotional experiences (Csikszentmihalyi \& Rathunde, 1993). Even simple manipulations such as increasing stimulus clarity and strength have a direct influence on positive emotionality (Reber, Winkielman, \& Schwarz, 1998). These findings motivate us to pay as much attention to warm as to cold factors in our measures of person perception.

A third theoretical motivation for exploring the potential of third-person measures stems from the interest of many researchers in studying cognition in the wild (Hutchins, 1995; Kingstone, Smilek, \& Eastwood, 2008; Tunnell, 1977). Recent research has responded by increasing the diversity of stimuli and expanding the variety of contexts in which cognition is studied. For example, depicted eyes and human limbs have replaced symbolic arrows in studies of attentional orienting (Nummenmaa \& Calder, 2009); photos and video clips have replaced line drawings in studies of face and scene perception (Henderson, 2005; Palermo \& Rhodes, 2007); and airport security and mammography xrays have replaced geometric shapes in studies of visual search (Wolfe, Horowitz, Van Wert, Kenner, \& Kibbi, 2007; Wolfe \& Van Wert, 2010). Although we see these trends as increasing the naturalism of cognitive research at the level of inputs and settings, we note that there has been less progress made in the measurement of the mind's output, as expressed in behavior. This is another reason we turn in this study to the question of whether person perception can augment our understanding of cognition.

To begin exploring the potential of person perception measures, we developed a two-phase research methodology in which standard performance measures (response time [RT] and accuracy) could be examined alongside measures of person perception that were obtained in an equally rigorous way. An overview of our research design and analyses is shown in Fig. 1. We will often return to this figure as a roadmap to our study, using it to show the relations between the various measures in the study and how we analyzed these measures and as a guide to the terminology we use.
In Phase 1 of the experiments, participants completed a standard visual search task, and we measured their performance. Participants in this phase were told that they were being videotaped for the purpose of "knowing where you looked while you were searching," but they were not informed that their videotaped performances would be used in Phase 2 of the research until after they had completed all trials in the search task. ${ }^{1}$ We expected from past research that search performance would be influenced by several factors (e.g., an individual's proficiency at visual search, the search strategy, and the difficulty of the search itself), and so we designed a task in which those influences could be isolated.

In Phase 2, a second sample of participants used their everyday person perception skills to observe and judge the behavior of Phase 1 participants, as seen in the video recorded searches. Each video corresponded to a trial in the search task of Phase 1, beginning with the onset of the stimulus display (which only the Phase 1 participant could see) and ending with the Phase 1 participant's responding where the target was located in the display. A random sample of each searcher's video clips was presented in a random order. Thus, both the experimenter and the Phase 2 participants were blind to the conditions under which the search behavior shown in the clips had been obtained.

We developed this methodology first for a study of visual search, because it is one of the most extensively studied tasks in all of cognitive psychology (Wolfe, 1998). As such, we already know much about how search is influenced by individual differences in search proficiency (Boot, Becic, \& Kramer, 2009), the adoption of cognitive strategies (Smilek, Dixon, \& Merikle, 2006a; Smilek, Enns, Eastwood, \& Merikle, 2006b; Watson, Brennan, Kingstone, \& Enns, 2010), and environmental factors that make search easy or difficult, including clutter (Smilek, Weiheimer, Kwan, Reynolds, \& Kingstone, 2009) and visual eccentricity (Wolfe, 1998). Search also has the advantage of occurring naturally in our everyday lives, whether it involves looking for car keys or locating a book on a shelf. As such, it is a cognitive task for which we are likely lifetime experts (Beck, Levin, \& Angelone, 2007; Levin \& Beck, 2004).

We began in Experiment 1 by having the Phase 1 participants search for common objects, depicted in a natural scene, while they were seated in front of a computer screen with their hands on the keyboard. This is typical of how visual search has been studied in the laboratory, and as such, it provided us with hundreds of potential search trials to sample from. However, it also left the participants with a limited range of motion, as compared with search under

\footnotetext{
${ }^{1}$ The UBC Behavioral Research Ethics Board approved this minor deception.
} 
Fig. 1 Overview of the research design and data analyses, showing the relations between the various measures and how these measures were analyzed and a guide to terminology

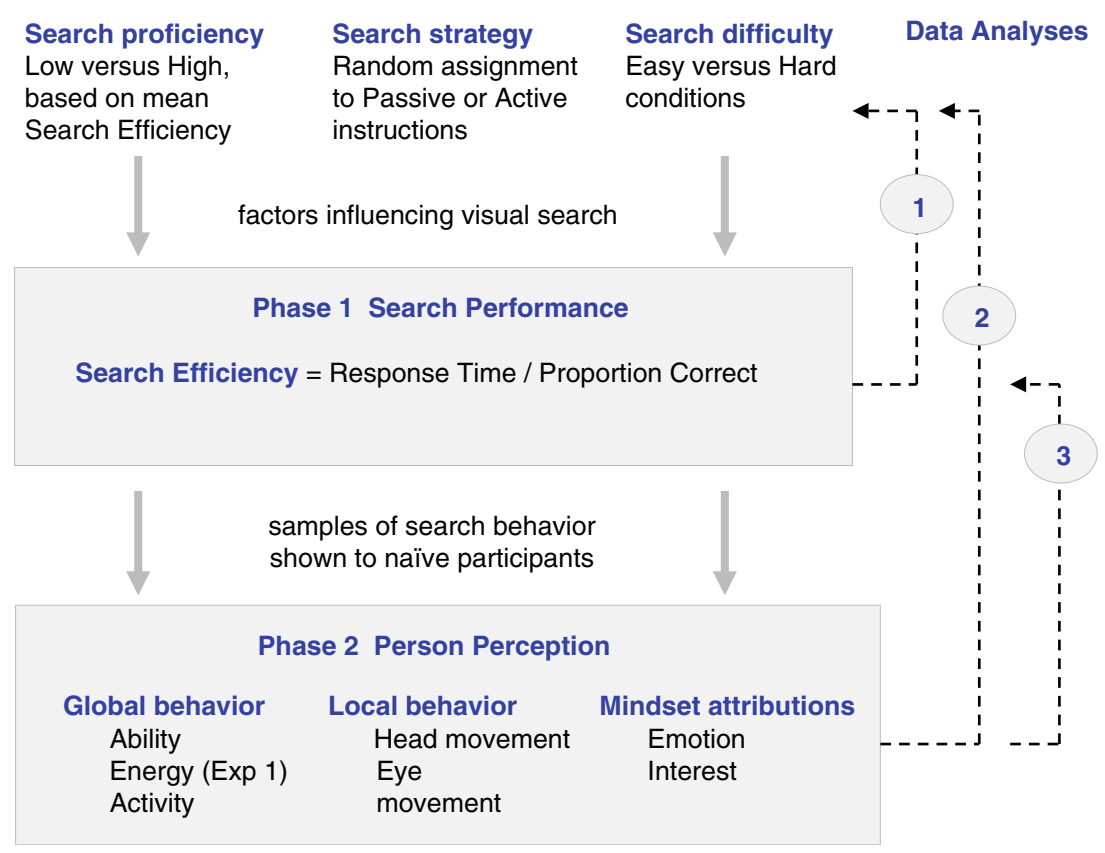

everyday conditions. The camera used to record participants' behavior was the iSight webcam on an iMac desktop computer, providing the views of search behavior illustrated in Fig. 2a.

In Experiment 2 we ventured out from this standard computer-based search task to examine visual search for items hidden in the actual office that had been depicted in Experiment 1. In Experiment 2, practical considerations reduced the opportunity to acquire a large number of trials as in Experiment 1, but at the same time, the search behavior we were able to record for person perception in Phase 2 was potentially richer. Each video clip, recorded with a camcorder positioned in a corner of the office, showed a Phase 1 participant entering a room and then scanning it in order to locate the target object, as illustrated in Fig. 2b. Participants indicated that they had found the target by raising their arm to point to its location.

\section{Experiment 1: Visual search on a computer screen}

The goal of the first experiment was to investigate the merits of person perception alongside performance measures of visual search. Specifically, we asked the following.

1. Is person perception sensitive to the same factors as performance measures of visual search - that is, to the influence of individual differences in proficiency, strategy, and environmental factors? As is shown in Fig. 1, this corresponds to asking whether the data analyses labeled 2 are sensitive to the same factors influencing search as the data analyses labeled 1.
2. How does person perception compare in its sensitivity to these factors with performance measures? This question is addressed by directly comparing analyses 1 and 2.

3. Which person perception ratings best predict search performance? This question is addressed by analysis 3 in Fig. 1, which asks to what extent the performance measures in Phase 1 can be predicted by the person perception measures in Phase 2.

Our aim in pursuing these questions was to determine whether research on human cognition could harness the person perception expertise of ordinary people, making judgments in real time. Directly comparing the sensitivity of person perception and performance measures on factors known to influence search (i.e., differences in individual proficiency, strategy use, and task difficulty) is an objective way to determine their validity. Finally, in selecting our person perception measures, we remained open to the possibility that indices of emotion and motivation might be as important as indices of the speed and accuracy of human movement.

The search task in Phase 1 was designed to result in sizable performance differences that could be attributed to individual differences in search proficiency, search strategy, and search difficulty. The strategy manipulation involved instructing a random one half of the participants to adopt an active search strategy, whereas the other one half of the participants were instructed to adopt a passive search strategy. This search strategy manipulation has been shown to create a passive advantage in search, whereby passive searchers are more efficient than active searchers (Smilek et al., 2006a, 2006b; Watson et al., 
Fig. 2 Representative still frame from a video of participants searching a on a computer screen in Experiment 1 and $\mathbf{b}$ in a real office in Experiment 2. Actors are posed in order to respect the privacy of the study participants
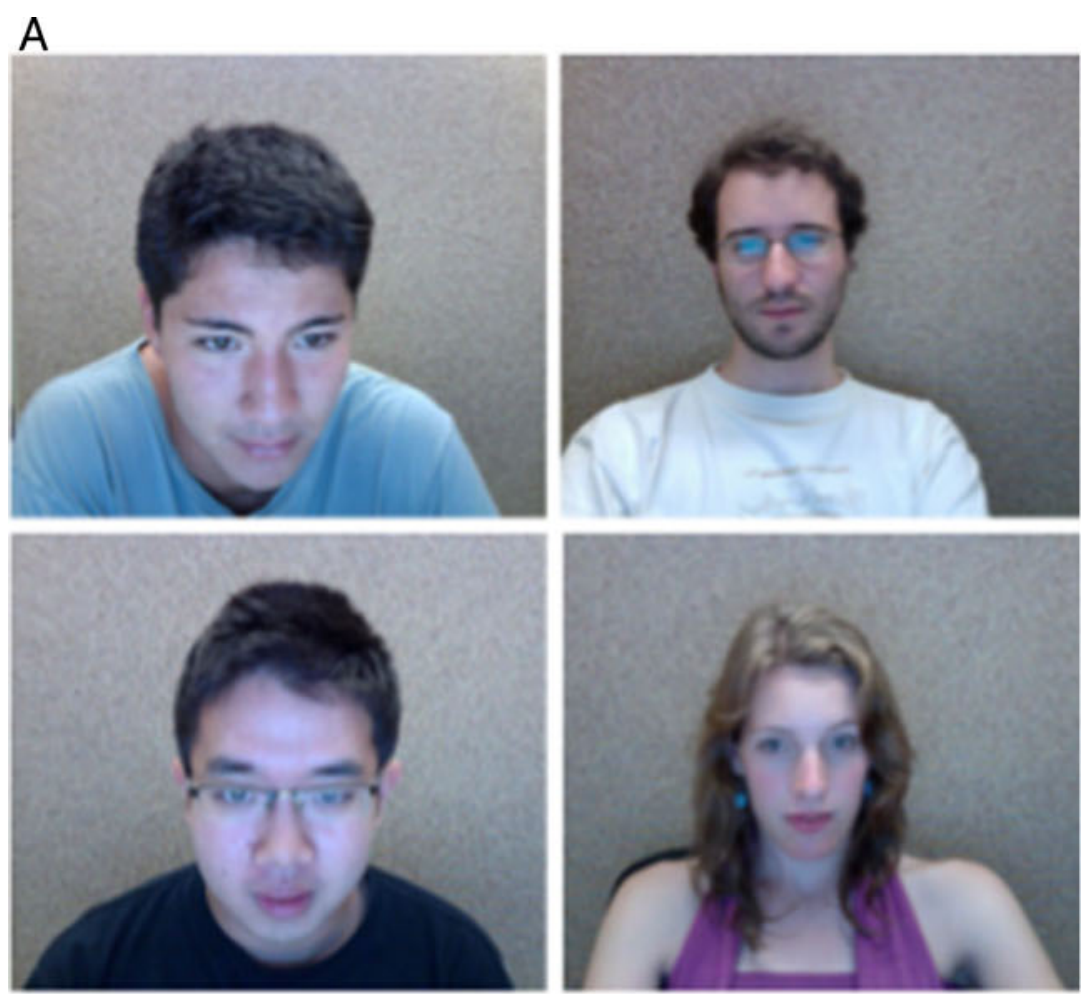

\section{B}
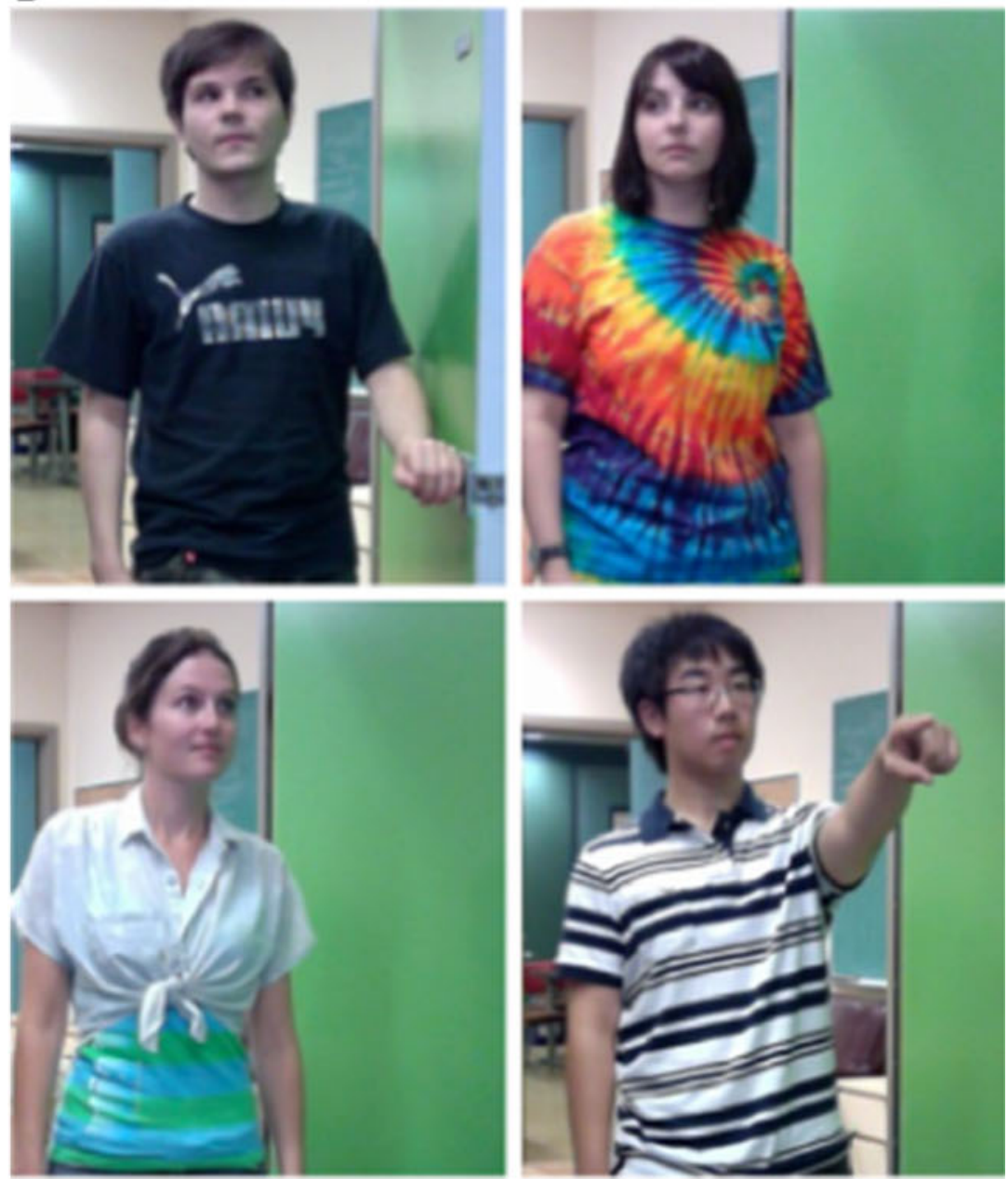

照 Springer 
2010). The search proficiency factor was participant defined, and it involved dividing participants in each of the two strategy groups into searchers of low and high proficiency on the basis of a median split of their average RTs in the search task. Finally, 10 different target objects were combined with eight different locations in the scene to create search conditions that varied over a wide range, from being generally easy for all participants to find to those that were generally hard.

The potential list of person perception measures for Phase 2 in this experiment is vast, and as such, we began by sampling items that ranged from those involving relatively global attributions about the behavior of the whole person, to ratings that were more narrowly focused on objectively defined behaviors, to ratings that required making attributions about the internal mental and emotional states of the participant. For convenience, we will refer to these classes as global behavior, local behavior, and mind-set attributions.

Among the ratings of global behavior were ability (defined as how fast and accurate a searcher appeared to be), energy (defined as how much physical effort was displayed by the searcher), and activity (defined as how much the searcher appeared to be searching actively, as opposed to passively). Ability and energy were assessed using a 6-point scale (with 6 representing maximum values), and activity was assessed with a 4-point scale. ${ }^{2}$

The ratings of local behavior included the frequency of head movements and the frequency of eye movements. Eye movements were included because of the previously established links between eye activity and success when a wide field of view was searched (Boot et al., 2009). The head movement scale was included because of the possibility that search on a relatively large display (24 in.) would require more head movements than have been typical of past experiments on smaller screens. These were both assessed using a 6-point scale (with 6 representing the maximum).

The ratings of mind-set attributions included emotion (defined as how much positive emotion the participant displayed upon finding the target) and interest (defined as how interested and engaged the participant appeared to be in the task). Positive emotions have been previously linked to successful performance in a number of cognitive tasks (Ashby, Isen, \& Turken, 1999; Gray, 2001, 2004; Phillips,

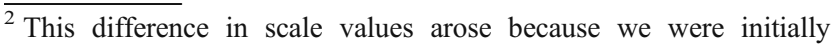
interested in whether Phase 2 participants could infer from visible behavior which instructions the Phase 1 participants had been assigned to, and so we designed a 4-point activity scale to index this $(1=$ confidently passive, $2=$ guess passive, $3=$ guess active, $4=$ confidently active).
}

Bull, Adams, \& Fraser, 2002), although not, to our knowledge, in visual search. Similarly, interest and engagement have been linked theoretically and empirically to improved cognition (Kane et al., 2007; Silvia, 2008). These were also assessed using a 6-point scale (with 6 representing the maximum).

\section{Method for Phase 1: Visual search}

Participants Twenty-four undergraduates (20 of them female) received course credit for participating in a session lasting less than $1 \mathrm{~h}$. All gave written informed consent to our initial description of the procedure. This description involved a minor deception, initially telling participants that the camera would record where they were looking and then revealing only at the conclusion of the search task that our intention was to use these recordings for person perception ratings. No one withdrew consent after we revealed this deception.

Stimuli and apparatus Search displays consisted of 80 photos of the cluttered office shown in Fig. 3a, presented on a 24-in. iMac computer. SuperLab4 software was used to randomize the trials and to collect the keyboard responses of the participants. The scene encompassed almost $40^{\circ}$ (horizontal) $\times 32^{\circ}$ (vertical) of visual angle, and the target objects each subtended less than $1^{\circ}$ of visual angle. The built-in iSight webcam of the iMac recorded video $(1,280 \times$ 1,024 pixel resolution) of the participants' upper body and head continuously throughout each testing session.

One of 10 common target objects, depicted in Fig. 3b, was present in each photo (keys, tea, pill bottle, milk, chalk eraser, mug, hole punch, box, staple remover, tape) in one of eight different locations. The locations for each object were determined by the orthogonal combination of four possible quadrants of visual space (relative to the center of the image) and two eccentricities (near the image center, in the periphery). As a result, targets appeared at both eccentricities in each quadrant, for eight unique locations per target object in total.

Procedure Each participant was tested in a single session, consisting of three randomized blocks of the 80 unique photos, for a total of 240 trials. To familiarize participants with the photos of the office and the target objects, each session began with participants viewing a screen with images of all 10 of the target objects for $30 \mathrm{~s}$. Participants then viewed a photo of the cluttered office without any of the target objects in it, but overlaid with a white grid indicating the numbered quadrants of visual space. While viewing this image, participants were asked to personally note key features of the cluttered scene, so that they would be able to retain these divisions of space once the grid lines were removed for the search test. Participants then practiced 
Fig. 3 a The cluttered office photograph searched by Phase 1 participants (Experiment 1) and $\mathbf{b}$ the 10 target objects

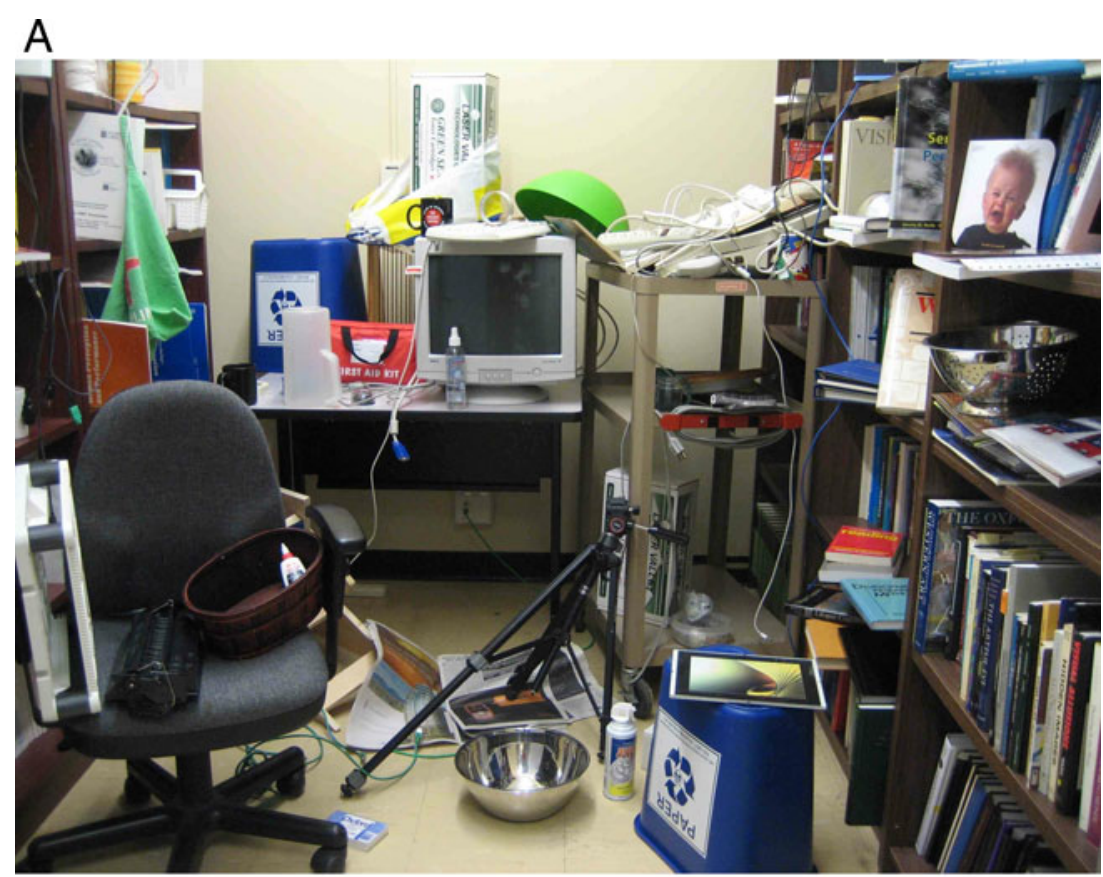

B

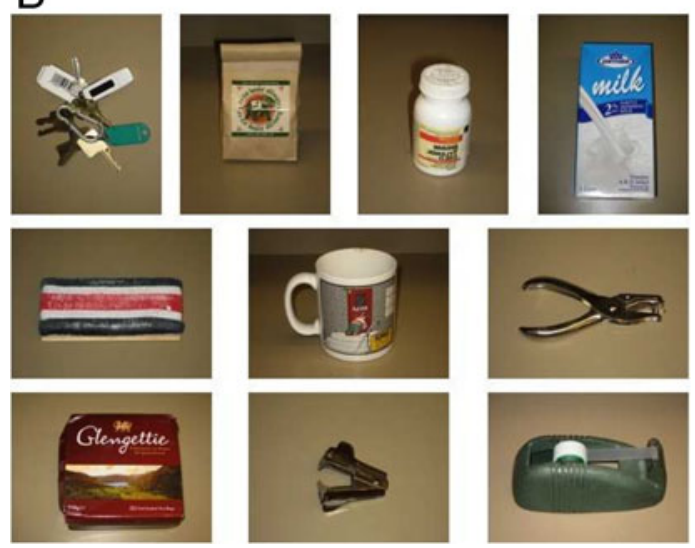

on 10 trials randomly selected from the larger set. Their instructions were to indicate the location of the target on each trail, as quickly and accurately as possible, by pressing one of four keys labeled 1-4, spatially mapped to correspond to the four quadrants on the screen.

On each trial, participants first saw a photo of one of the 10 target objects, displayed in the center of the screen for $2 \mathrm{~s}$. The office photo containing the target in one of its eight possible locations was then shown until participants responded with a keypress or until $15 \mathrm{~s}$ had elapsed (also recorded as an error).

The only factor that was systematically varied between participants was the instruction concerning cognitive strategy (Smilek et al., 2006b, Watson et al., 2010). A random half of the participants were assigned to the active group and were told the following:

The best strategy for this task, and the one that we want you to use in this study, is to be as active as possible and to "search" for the target as you look at the screen. The idea is to deliberately direct your attention to determine your response. Sometimes people find it difficult or strange to direct their attention-but we would like you to try your best. Try to respond as quickly and accurately as you can while using this strategy. Remember, it is very critical for this experiment that you actively search for the target.

The other half of participants were assigned to the passive group and were told the following:

The best strategy for this task, and the one that we want you to use in this study, is to be as receptive as possible and let the target "pop" into your mind as you look at the screen. The idea is to let the display and your intuition determine your response. Sometimes people find it difficult or strange to tune into their gut feelings - but we would like you to try your best. Try 
to respond as quickly and accurately as you can while using this strategy. Remember, it is very critical for this experiment that you let the target just "pop" into your mind.

Method for Phase 2: Person perception of search behavior

Participants Sixty-nine undergraduates (52 of them female) received course credit for participating in a half-hour session. These participants were instructed to rate the visible behavior of the Phase 1 searchers along the following dimensions: global behavior, ability $(\mathrm{n}=11)$, energy $(\mathrm{n}=9)$, and activity $(\mathrm{n}=10)$; local behavior, head movement $(\mathrm{n}=10)$ and eye movement $(\mathrm{n}=10)$; and mind-set attributions, positive emotion $(n=9)$ and interest $(n=10)$. See the Appendix for the complete rating instructions.

Stimuli and apparatus Phase 1 yielded a total of 5,760 video clips of search trials in total (24 participants $\times 240$ search trials). After excluding video clips of searches longer than $13 \mathrm{~s}(n=56)$, we randomly sampled 8 video clips from each of 24 Phase 1 search participants, for a total of 192 video clips. The 8 clips were selected by sampling orthogonally across three dimensions: (1) task familiarity, where half of the clips were from the beginning of the session (trials 0-32), and half were from the end (trials 214-240); (2) target eccentricity, where half involved targets located near the center of the scene, and half involved targets located in the periphery; (3) task difficulty, where half of the clips were from trials defined as easy, and half were from trials defined as hard (based on the average search time of all Phase 1 participants). These video clips were edited so that they began when the search display appeared on the screen and ended when participants made their response indicating a target object location. Figure $2 \mathrm{a}$ shows representative frames from these videos. The video clips of hard searches (originally, $M=4,864 \mathrm{~ms}, S D=$ $558.34 \mathrm{~ms}$ ) were shortened to include only the last $4 \mathrm{~s}$ of the search, including the discovery of the target. Easy search clips were unedited ( $M=2,353 \mathrm{~ms}, S D=281.43 \mathrm{~ms}$ ). Because the lengths of the easy and hard video clips were not statistically equated, we performed an analysis of covariance to examine task difficulty after controlling for differences in length (see the Results and Discussion section).

Procedure Each participant was tested in a single session where he or she viewed a total of 192 video clips of Phase 1 participants in a random order (24 Phase 1 participants $\times 2$ levels of familiarity $\times 2$ levels of eccentricity $\times 2$ levels of search difficulty). The video clips were presented on a 24in. iMac computer, using SuperLab 4. In order to familiarize participants with the range of behavior depicted in the videos, participants practiced prior to being tested by rating 10 video clips selected at random from the entire set.

\section{Results and discussion}

Analysis 1: Search performance is sensitive to search proficiency, search strategy, and search difficulty Figure 4 shows the search performance of all participants as a function of these three factors. Search performance is summarized by way of an efficiency score, which is obtained by dividing correct RT by the proportion of correct responses (PC) for each participant in each condition. This score is a convenient way to combine search time and accuracy when they are related, as they are here, because it corrects RT values that are underestimated when participants are willing to trade errors for RT (Townsend \& Ashby, 1983; Watson et al., 2010).

Figure 4 demonstrates that each of these three factors had sizable influences on search efficiency, thus providing the appropriate context for the study of person perception in Phase 2. Table 1 gives details on the relative effect sizes for each of the factors. These results indicate, first, that actively instructed participants searched more efficiently than did those in the passive group, in contrast with previous reports that passive searchers are more efficient than active searchers (Smilek et al., 2006b; Watson et al., 2010). Second, within each of these

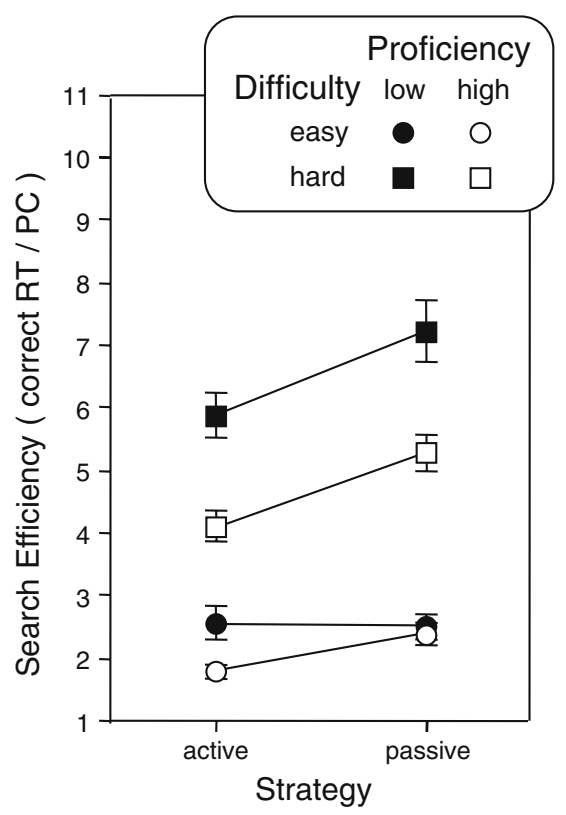

Fig. 4 Mean efficiency scores (correct response time/proportion correct) in Experiment 1 as a function of search strategy, search proficiency, and task difficulty. Error bars are \pm 1 standard error of the mean 
Table 1 Mean differences, standard errors, and two effect size measures (Cohen's $d$ and partial eta-squared) for the effects of proficiency, strategy, and difficulty in Phase 1 of Experiments 1 and 2

\begin{tabular}{llll}
\hline & Proficiency & Strategy & Difficulty \\
\hline Exp 1: Computer Screen & & & \\
Mean difference (seconds) & 1.146 & 0.771 & 3.314 \\
Pooled standard error & 0.286 & 0.296 & 0.172 \\
Cohen's $d$ & 0.566 & 0.373 & 2.59 \\
Partial eta-squared & .575 & .380 & .951 \\
Exp 2: Natural Setting & & & \\
Mean difference (seconds) & 3.34 & 1.84 & 4.26 \\
Pooled standard error & 0.699 & 0.746 & 0.632 \\
Cohen's $d$ & 0.906 & 0.468 & 1.25 \\
Partial eta-squared & .499 & .234 & .643 \\
\hline
\end{tabular}

Cohen's $d=$ (large mean - small mean) / pooled standard deviation, partial eta-squared $=$ SSeffect $/($ SSeffect + SS error $)$

two instruction groups, there were still sizable individual differences in search efficiency, such that a median split of the participants (i.e., low- and high-proficiency searchers) led to differences in search efficiency that were comparable in magnitude to the effects of strategy. Third, some target objects were generally more difficult to find than other target objects, regardless of where in the display they were located and regardless of the previously described individual differences. Finally, Fig. 4 shows that these three factors interacted, such that differences between strategies and proficiencies were most readily apparent when the search task was hard. These conclusions were based on the following statistical analyses.

In order to coalesce a sufficient amount of data so that stable interactions could be examined between the factors of search proficiency, strategy, and difficulty, we first simplified the difficulty factor by grouping the 10 target objects into two clusters. Seven of the objects were grouped into an easy condition (tea, pill bottle, milk, chalk eraser, mug, box, tape; mean score $=2,314$ ), and 3 objects were grouped into a hard condition (keys, hole punch, staple remover; mean score $=5,628$ ).

The two-level search difficulty factor (easy, hard) was then combined with the between-groups factors of search strategy (active, passive) and search proficiency (low, high) in a mixed-design analysis of variance (ANOVA) of the search efficiency scores. This analysis indicated a significant main effect of proficiency, $F(1,20)=12.26, p<.01$, a significant main effect of strategy, $F(1,20)=27.99, p<.01$, and a significant main effect of difficulty, $F(1,20)=392.87, p<$ .001 . In addition, there were significant two-way interactions between proficiency and difficulty, $F(1,20)=8.87, p<.01$, and strategy and difficulty, $F(1,20)=17.67, p<.01$. No other effects were significant (all $p \mathrm{~s}>.20$ ).
Analysis 2: Person perception ratings are sensitive to search proficiency, search strategy, and search difficulty, although to varying degrees In this section, we describe the sensitivity of person perception ratings to each of the factors influencing visual search, which is an important proof of the concept that person perception can be sensitive to the same factors to which performance measures are sensitive. However, not all ratings are equally sensitive. Specifically, we found that person perception ratings of searcher activity, eye movement frequency, interest, and positive emotion were more sensitive to individual differences between searchers than were ratings of searcher ability, a rating that seems, at face value, to ask about search performance even more directly. These conclusions were based on the following statistical analyses.

Interrater reliability The mean ratings of the video clips on each of the dimensions generally yielded high interrater agreement among Phase 2 participants (Cronbach's alpha for rated ability $=.672$, activity $=.920$, energy $=.882$, head $=$ .981 , eye $=.913$, emotion $=.912$, interest $=.881$ ). This strong agreement suggests that raters were reliably detecting and evaluating the behaviors we had instructed them to assess. We would like to note that ability was rated less reliably than the other dimensions, suggesting that there was less agreement in person perception ratings about what constitutes a good versus a poor searcher (where good is defined as fast and accurate) than about what constitutes physical activity or positive emotion.

Figure 5 shows the mean ratings for each of seven rated dimensions as a function of search proficiency (low, high) and search strategy (active, passive). The search difficulty factor, although not shown here in order to focus our analysis on individual differences in the ratings, also had an influence on many of the ratings, as described below.

Ratings of global behavior Phase 2 participants were most sensitive to the factors of proficiency, strategy, and difficulty when they were asked to rate how active searchers were. Ratings of overall activity were sensitive to all three factors [proficiency, $F(1,9)=59.89, p<.001$; strategy, $F(1,9)=69.65, p<.001$; difficulty, $F(1,9)=$ $5.86, p<.04]$, with greater levels of rated activity assigned to higher proficiency searchers, to active searchers, and to search in the more difficult conditions. With the influence of clip length controlled in an analysis of covariance, activity ratings remained sensitive to differences in search difficulty, $F(1,8)=15.84, p<.01$. Ratings of ability were less sensitive to the same three factors, showing only a marginally significant sensitivity to strategy, $F(1,10)=$ $4.68, p<.06$, and difficulty, $F(1,10)=3.39, p<.10$. Ratings of energy were sensitive only to the proficiency factor, $F(1,9)=17.44, p<.01$. 
Fig. 5 Mean ratings for the seven person perception dimensions in Experiment 1 as a function of search proficiency (low, high) and search strategy (active, passive). Error bars are \pm 1 standard error of the mean
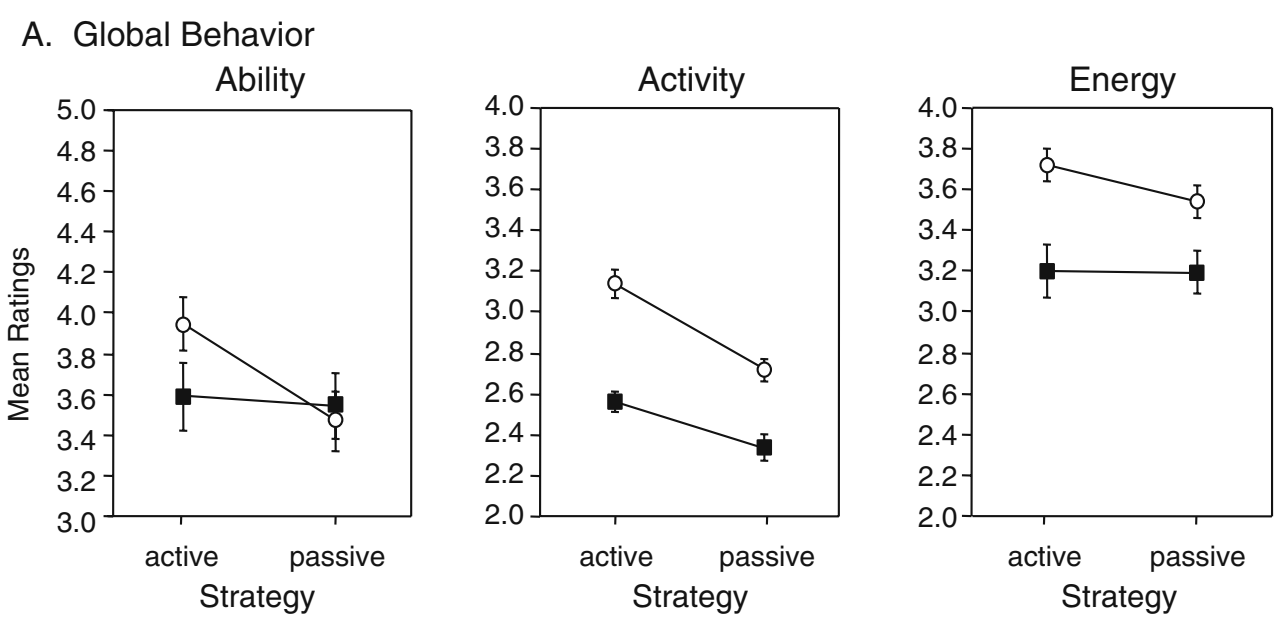

B. Local Behavior

Search Proficiency
$\circ$ High
- Low

B. Mindset Attribution
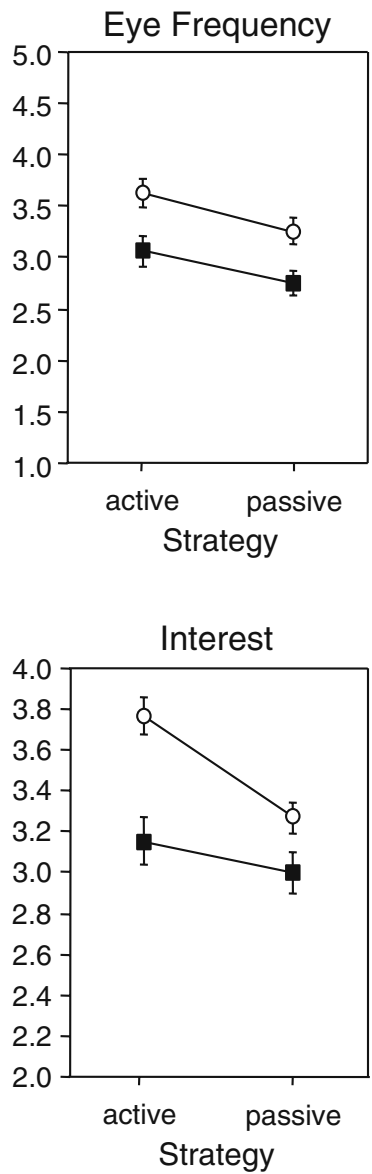

Strategy
Ratings of local behavior Ratings of head movement frequency were sensitive to all three factors [proficiency, $F(1,8)=$ 214.74, $p<.001$; strategy, $F(1,8)=17.55, p<.01$; difficulty, $F(1,8)=132.67, p<.001]$. With the influence of clip length controlled in an analysis of covariance, head movement frequency ratings remained sensitive to differences in search difficulty, $F(1,7)=101.00, p<.01$. Ratings of eye movement frequency were sensitive to the factors of proficiency, $F(1,9)=$ 75.26, $p<.001$, and strategy, $F(1,9)=17.25, p<.01$.
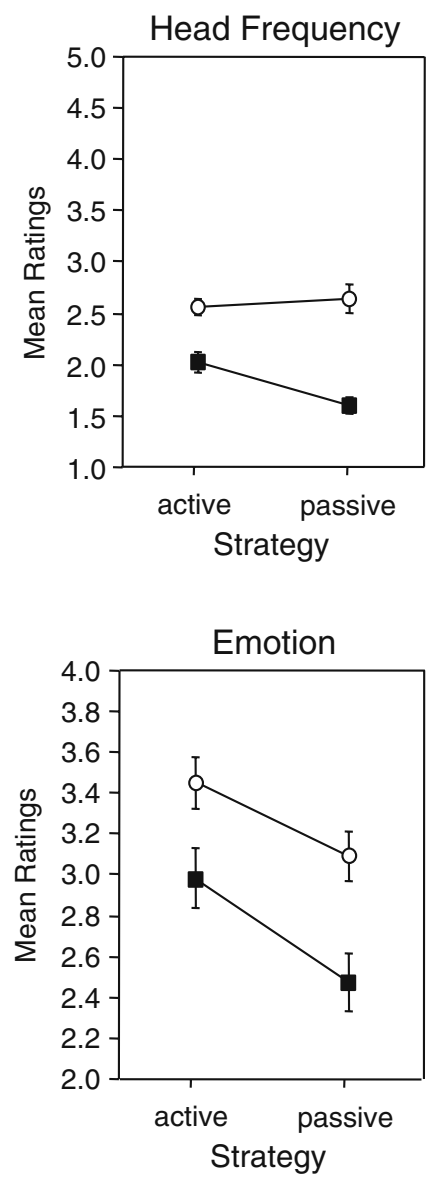

Mind-set attribution ratings Rating of searchers' emotion upon detection of the target was sensitive to all three factors [proficiency, $F(1,9)=60.60, p<.001$; strategy, $F(1,9)=$ $22.59, p<.01$; difficulty, $F(1,9)=16.12, p<.01]$. With the influence of clip length controlled in an analysis of covariance, emotion ratings remained sensitive to differences in search difficulty, $F(1,8)=22.76, p<.01$. Ratings of interest were sensitive to the factors of proficiency, $F(1,9)=$ 20.22, $p<.001$, and strategy, $F(1,9)=33.90, p<.01$. 
Table 2 shows the outcome of a head-to-head comparison between the performance measure of search efficiency in Phase 1 and the seven different person perception ratings in Phase 2. The comparison was based on multiple regression analyses, where for each measure, we examined the partial correlation $(r)$ for the predictors of proficiency (high, low) and strategy (active, passive), along with the total variance explained by these two factors $\left(R^{2}\right)$. The unit of prediction was the mean efficiency score for each of the 24 participants in the hard search conditions, where individual differences in the performance of the searchers were the greatest.

The last column in Table 2 indicates the extent to which variance among individual searchers is explained by the factors of proficiency and strategy. It shows that the performance measure (i.e., search efficiency) accounted for $68.5 \%$ of the variance and that this measure was significantly sensitive to individual differences arising from both proficiency, $r=.684, t(21)=5.58, p<.001$, and strategy, $r=.465, t(21)=3.80, p<.001$.

Among the global attribution ratings, activity was the most sensitive rating scale, accounting for $48.8 \%$ of the variance and also showing independent sensitivity to proficiency, $r=.581, t(21)=3.72, p<.01$, and strategy, $r=.387, t(21)=2.48, p<.02$. Both ability ratings and energy ratings were less sensitive, accounting for $27.7 \%$

Table 2 Multiple regression analyses of individual differences in proficiency (based on a median split of search efficiency in each strategy group) and strategy (based on the random assignment of participants to either an active or a passive strategy condition) as predicted by search efficiency and by the seven third-person ratings in Phase 2 of Experiment 1. The data include all 24 searchers and are taken from trials in the hard search condition, where individual differences were the greatest

\begin{tabular}{|c|c|c|c|}
\hline Measure & Proficiency $r$ & Strategy $r$ & $R^{2}$ \\
\hline \multicolumn{4}{|c|}{ Phase 1 Performance: } \\
\hline Search efficiency & $.684 * * *$ & $.465^{* * *}$ & $.685^{* * *}$ \\
\hline \multicolumn{4}{|c|}{ Phase 2 Global Behavior Ratings } \\
\hline Ability & $.362 *$ & $.381 * *$ & $.277 * *$ \\
\hline Activity & $.581 * * *$ & $.387 * *$ & $.488^{* * *}$ \\
\hline Energy & $.464 * *$ & .029 & $.216^{*}$ \\
\hline \multicolumn{4}{|c|}{ Phase 2 Local Behavior Ratings } \\
\hline Head movement & .296 & .050 & .090 \\
\hline Eye movement & $.332 *$ & $.436 * *$ & $.300^{* *}$ \\
\hline \multicolumn{4}{|c|}{ Phase 2 Mindset Ratings } \\
\hline Emotion & $.429 * *$ & $.384 * *$ & $.332 * * *$ \\
\hline Interest & $.498 * * *$ & .279 & $.326^{* *}$ \\
\hline
\end{tabular}

and $21.6 \%$ of the variance, respectively, with ability ratings showing significant specific sensitivity to strategy and energy ratings showing a specific sensitivity to proficiency.

Among the local behavioral ratings, frequency of eye movements $(30.0 \%$ of variance explained) showed greater sensitivity to individual differences in performance than did frequency of head movements ( $9.0 \%$ of variance explained).

Finally, the two ratings of mind-set also showed some sensitivity, with variance shared between efficiency scores and emotion ratings equal to $33.2 \%$ and between efficiency scores and interest ratings equal to $32.6 \%$.

Perhaps the most interesting of these findings was that ratings of searcher activity and of eye movement frequency were more sensitive to the individual differences between searchers than were ratings of searcher ability. This is of importance because ratings of activity and eye movement frequency are relatively indirect measures of the psychological construct under investigation (i.e., the ability to search efficiently), whereas the rating of searcher ability is a direct measure. Indeed, folk wisdom suggests that direct measures should trump indirect measures of psychological states. In the case of person perception, this translates to "just look at them and see." However, the social psychology literature also warns against this folk wisdom. In many cases, the measurement of a person's attitude that best predicts their behavior is done using an implicit test. By contrast, an explicit test is more vulnerable to the influence of experimenter demand (Orne, 1962 ) and socially appropriate responding (Greenwald, Poehlman, Uhlmann, \& Banaji, 2009; Maison, Greenwald, \& Bruin, 2004) and to the limitations of consciously accessible decision processes (Dijksterhuis, 2004). Having observed the greater reliability and validity of indirect measurement of search ability in person perception ratings, it will be important in future research to determine which visible behaviors underlie these less reliable ratings of search ability.

Analysis 3: The person perception ratings that best predict search performance are eye movement activity and positive emotion In these analyses, we addressed the question of which person perception ratings, either alone or in combination, did the best job of accounting for variance in the detailed search performance data of Phase 1. Because some of these ratings scales were also correlated strongly with one another, we used a simultaneous regression procedure to help determine which rating scales contained the greatest amount of unique information with regard to search performance. This analysis indicated that over $63 \%$ of all the variance in the performance of individual searchers could be accounted for by just two rated variables: the frequency of eye movements and expressions of positive emotion in the faces of searchers. 
Table 3 shows cross-correlations for all person perception scales. Clearly, there is much overlap in the information contained in these ratings, with, for example, activity ratings and eye movement frequency ratings having much in common $(r=.773)$ and emotion and interest ratings sharing a great deal of variance $(r=.843)$. In contrast, eye movement and head movement frequency are relatively uncorrelated $(r=.119)$.

Table 4 shows the results of our regression analyses. The first column shows simple correlations between mean individual search efficiency scores and the seven person perception ratings. To see which of these ratings contributed uniquely to the individual differences in search efficiency, we entered all of them as predictors into a simultaneous multiple regression model in which the efficiency scores of the 24 searchers represented the outcome variable. The full model, involving all seven ratings, yielded an $R^{2}$ value of $.635, F(7,16)=3.97$, $p<.01$. By systematically removing ratings that contributed least to the total variance explained, as indicated by their partial coefficients, we found that a reduced model involving only two of the ratings, frequency of eye movements and emotion upon finding the target, still accounted for a similar amount of variance, $R^{2}=.608$, $F(2,21)=16.31, p<.001$. The partial coefficients for each of these ratings were significant $(p<.01)$, indicating that they each contributed significantly as predictors beyond the simple correlation involving only one of the predictors.

\section{Experiment 2: Visual search in a natural setting}

In Experiment 1, we demonstrated the merits of person perception measures to inform understandings of visual search in a cluttered office scene. We found that some thirdperson ratings of search behavior were almost as sensitive as the performance measures in Phase 1 to the factors influencing search (i.e., proficiency, strategy, and difficulty). Specifically, we found that indirect ratings of searcher activity and of eye movement frequency were more sensitive (i.e., better detected the influence of the factors influencing search) to individual differences among searchers than were more direct ratings of searcher ability. In addition, we uncovered a relationship between ratings of positive emotional expression and search efficiency with the use of person perception measures; participants expressing more positive emotion upon finding the target were more efficient at visual search.

With Experiment 2, our aim was to extend these findings to a less constrained environment - namely, to the actual office setting that was depicted in Experiment 1. We were motivated by the possibility that person perception in a more natural setting might be richer than that in a conventional laboratory search on a computer screen. However, before studying person perception in this setting, we first had to establish that search would still be influenced by the same factors as in Experiment 1 (i.e., proficiency, strategy, difficulty). Therefore, as before, the search task in Phase 1 of Experiment 2 was intended to result in sizable individual differences in search strategy (defined by instructions to participants to adopt either an active or a passive cognitive search strategy) and search proficiency (defined by dividing participants in each of the two strategy groups into low- and high-proficiency searchers, on the basis of a median split of their average search efficiency scores) and to establish that environmental factors would influence search difficulty (different target objects were combined with different locations to create search conditions that ranged from generally easy to hard).

Designing a natural setting visual search task also prompted several practical considerations. For example, because actual target objects had to be relocated in the office between trials, it was no longer possible to test hundreds of unique trials, as in Experiment 1. Instead, only five different target objects were combined with three unique locations to generate 15 different search trials in total. Second, a trial no longer began with the sudden onset of a computer picture and ended with a keypress. Instead, it began when the participant first opened the door to the office and ended when the participant raised an arm to indicate the location of the target. Third, the search environment of the cluttered office occupied participants' entire visual field (as compared with the computer scene in Experiment 1, which occupied $40^{\circ}$ [horizontal] $\times 32^{\circ}$
Table 3 Correlations among the seven third-person rating scales in Phase 2 of Experiment 1

$* \mathrm{p}<.05$

$* * \mathrm{p}<.01$

\begin{tabular}{|c|c|c|c|c|c|c|c|}
\hline & Ability & Activity & Energy & Head & Eyes & Emotion & Interest \\
\hline Ability & & $.460 *$ & $.643 * *$ & .397 & .161 & $.683^{* *}$ & $.694 * *$ \\
\hline Activity & & & $.653 * *$ & $.421 *$ & $.773 * *$ & $.566^{* *}$ & $.667 * *$ \\
\hline Energy & & & & $.509^{*}$ & .285 & $.680 * *$ & $.781 * *$ \\
\hline Head & & & & & .119 & $.790 * *$ & $.664 * *$ \\
\hline Eyes & & & & & & .197 & .320 \\
\hline Emotion & & & & & & & $.843 * *$ \\
\hline Interest & & & & & & & \\
\hline
\end{tabular}


Table 4 Multiple regression models using person perception ratings to predict individual differences in search efficiency in Experiment 1

\begin{tabular}{llll}
\hline Ratings & Simple $r$ & $\begin{array}{l}\text { Full Model } \\
\text { Partial } r\end{array}$ & $\begin{array}{l}\text { Reduced Model } \\
\text { Partial } r\end{array}$ \\
\hline Ability & $.480^{* *}$ & .104 & \\
Activity & $.587^{* *}$ & .096 & \\
Energy & $.425^{* *}$ & .155 & \\
Head & $.543^{* * *}$ & .074 & $.518^{* * *}$ \\
Eyes & $.506^{* *}$ & $.414^{*}$ & $.688^{* * *}$ \\
Emotion & $.682^{* * *}$ & $.398^{*}$ & \\
Interest & $.600^{* *}$ & .007 & .608 \\
& & & \\
$R^{2}$ & & .635 & \\
\hline
\end{tabular}

The first column shows simple correlations between mean individual search efficiency scores and the seven ratings. Asterisks refer to statistical significance of these correlations: ${ }^{*} p<.10,{ }^{* *} p<.05,{ }^{* *} p<.01$. Partial $r$ is a relative measure of unique variation for each predictor; $R^{2}$ is the proportion of variance in the individual search efficiency that is accounted for by the predictors in each model

[vertical] of visual angle). Aside from these practical considerations, we conducted the search task and the person perception measures in the same way as in Experiment 1.

\section{Method for Phase 1: Visual search}

Participants Twenty-four undergraduates (12 of them female) received course credit for participating in a half-hour session. All gave written informed consent and were treated in accordance with APA standards. As in Experiment 1, no one withdrew their consent when they were asked whether their videotapes could be used in Phase 2 of the research.

Procedure Figure 6a shows five common objects (book, hole punch, keys, mug, and pill bottle) that were each hidden in a cluttered office (see Fig. 6b) at three different locations. Each target object was visible to the participant once the door to the office door was opened but appeared in a different location on each occasion (see Fig. 6c). Participants always stood on a location indicated by tape on the floor positioned $30 \mathrm{~cm}$ from the doorway. A digital video camera (image resolution, $1,024 \times 768)$ in the right corner of the office captured the upper body and head of the participant. Participants were informed that they would be video recorded in order to determine where they were looking while searching.

Each participant was tested in a single session involving 15 trials in random order. Prior to testing, participants viewed the target objects from various angles in order to become familiar with them. All participants were instructed to find the target as quickly as possible. In addition, 12 participants were instructed to actively direct their attention in search of the target; the other 12 were instructed to passively search for the target (e.g., let the target pop into mind), as was done in Experiment 1 (and following Smilek et al., 2006b, Watson et al., 2010), with two alterations: "as you look around the room" (in place of "as you look at the screen") and "to find the target" (in place of "to determine your response").

During testing, a trial began with the experimenter displaying the target, before going into the office and placing it in the scene with the door closed. Visual search timing was based on the video record of each trial, with the time beginning when the door to the office opened and ending when the participants raised their arm to point at the target.

Method for Phase 2: Person perception of search behavior

Participants Fifty-nine undergraduates (45 of them female) received course credit for participating in a half-hour session. Participants rated the search behavior of Phase 1 participants along the same dimensions as in Experiment 1: global behavior, ability $(\mathrm{n}=10)$, activity $(\mathrm{n}=10)$; local behavior, head movement $(\mathrm{n}=7)$, eye movement $(\mathrm{n}=7)$; and mind-set attributions, positive emotion $(\mathrm{n}=7)$, interest $(\mathrm{n}=10)$.

Stimuli Phase 1 yielded a total of 360 video clips (24 participants $\times 15$ search trials). After excluding searches longer than $25 \mathrm{~s}(n=33)$, we randomly sampled 4 video clips from each of 24 Phase 1 search participants, for a total of 96 video clips. Half of the clips were from trials defined as easy; half were from trials defined as hard (on the basis of the average search time of all Phase 1 participants). Clips of easy searches were edited to begin with the door opening and end with the participant's pointing to the target (see Fig. 2b), while clips of difficult searches (originally, $M=$ $9.08 \mathrm{~s}, S D=4.54 \mathrm{~s}$ ) were clipped to include only the final $5 \mathrm{~s}$ of search, including the discovery of the target, so that they were approximately equal to the length and variability of the easy searches $(M=4.82 \mathrm{~s}, S D=1.64 \mathrm{~s})$.

Procedure Each participant was tested in a single session where they viewed the 96 video clips of Phase 1 participants' search behavior, described above, in a randomized order. The experiment was presented to Phase 2 participants on a 24-in. iMac computer, using SuperLab 4. Participants completed 10 practice ratings, selected at random, to become familiar with the full range of behavior exhibited by Phase 1 search participants before beginning the experiment. Participants were instructed to rate the behavior of previous participants who were searching for common objects in a cluttered office. If judging whether Phase 1 participants had been instructed to search either actively or passively, participants read both sets of instructions prior to the start of the experiment. 
Fig. 6 a The five target objects in Phase 1 (Experiment 2), b the participant's view of the cluttered office, and $\mathbf{c}$ a schematic indicating the location of 12 target objects (green $=$ easy, blue $=$ hard). The video camera can be seen just below the righthand side picture on the far wall
A
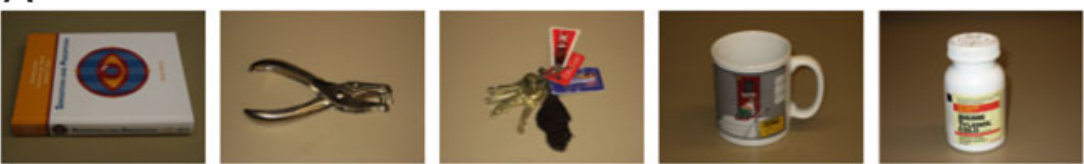

B

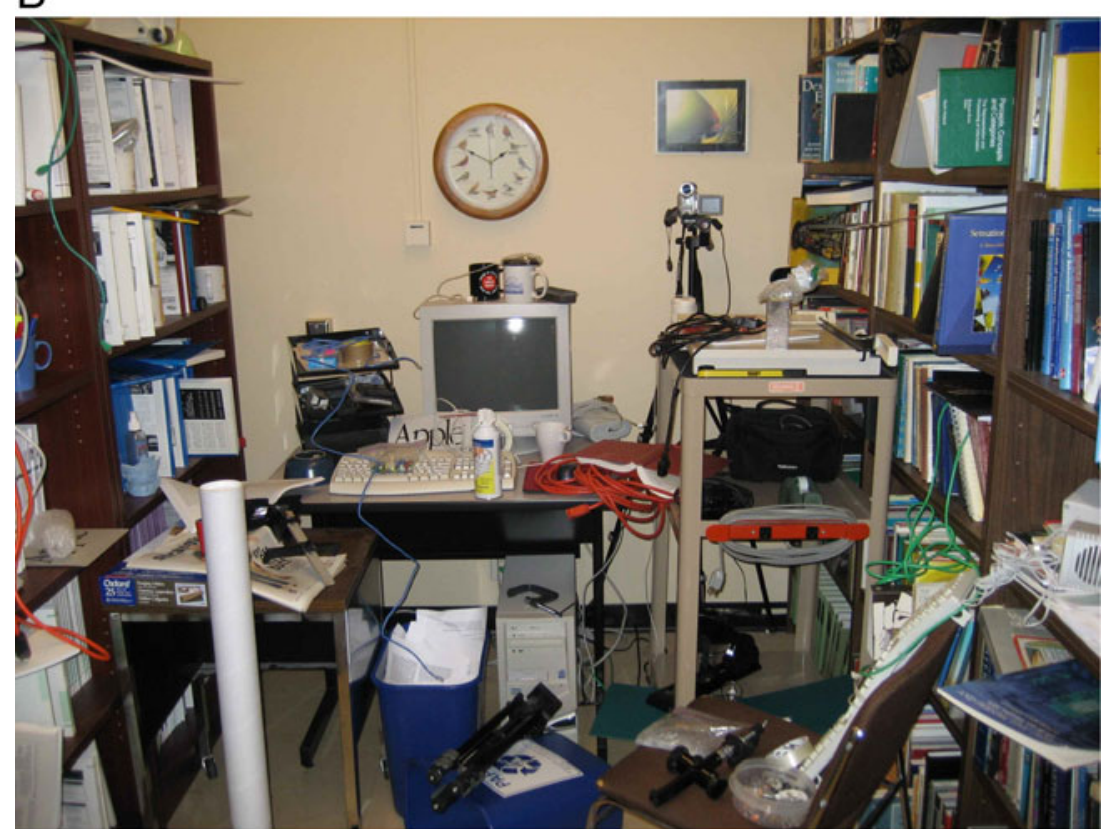

C

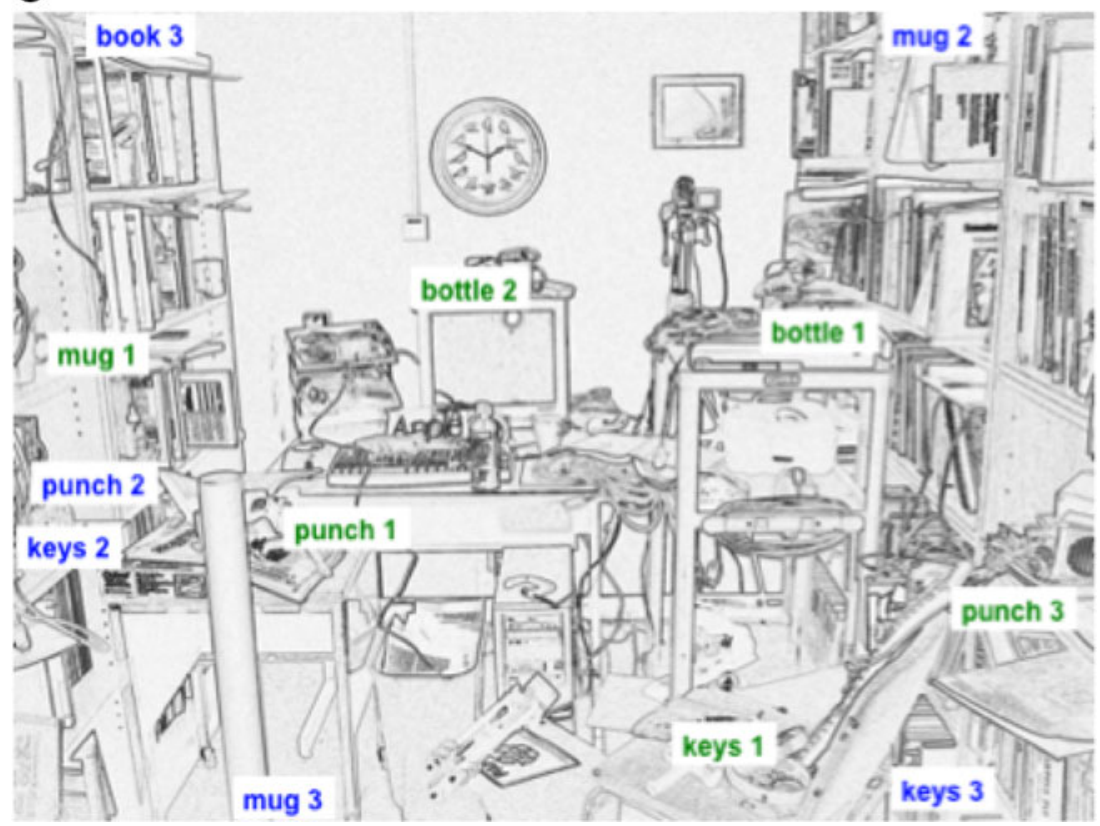

Phase 2 participants made local, global, and mind-set ratings using the same 4- and 6-point scales as in Experiment 1 . However, we decided to omit energy ratings in Experiment 2 because results showed that they were so closely related to activity ratings in Experiment 1 . See the Appendix for the complete rating instructions.

\section{Results and discussion}

Analysis 1: Natural setting search performance is sensitive to search proficiency, search strategy, and search difficulty The purpose of these analyses was to establish that search in the actual cluttered office was sensitive to the 
same factors shown to be important in computer screen searches. Figure 7 shows the search performance of all participants as a function of search proficiency, search strategy, and search difficulty. Performance was summarized here, as in Experiment 1, with an efficiency score obtained by dividing correct RT by PC for each participant in each condition, which in this experiment was 1.0 because the target was always found.

Figure 7 shows that the three factors had sizable influences on search performance, and Table 1 gives details on their relative effect sizes. As in Experiment 1, the assignment of participants to either an active or a passive search strategy resulted in active searchers being generally faster. Second, there were individual differences in efficiency within each strategy group, similar in magnitude to the effects of adopting a search strategy, allowing us to again categorize participants as either low- or highproficiency searchers. Finally, some searches were generally more difficult than others for all participants, allowing us to once again categorize the results on the basis of search difficulty. These conclusions were based on the following analyses.

A preliminary inspection of the data indicated that 3 of the 15 search trials had large and variable efficiencyscores $(M=15.91, S D=7.09)$. They involved targets that were located on the extreme perimeter of the office, such that their view was occluded when the door was not fully

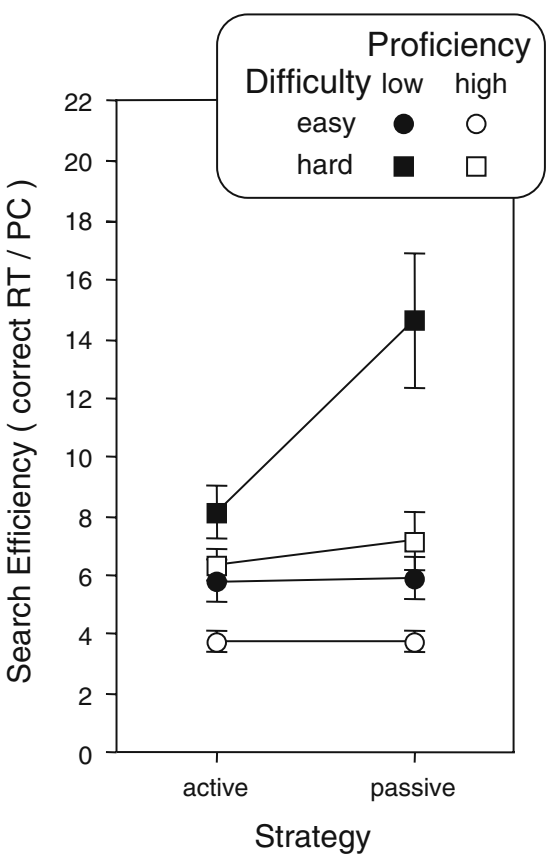

Fig. 7 Mean search time in Experiment 2 as a function of search strategy, search proficiency, and task difficulty. Error bars are \pm 1 standard error of the mean open. We therefore excluded these 3 trials, although including them did not influence the overall pattern of results. The remaining data were examined in a mixeddesign ANOVA involving the repeated measures factor of search difficulty (easy, hard) and the between-participants factors of search strategy (active, passive) and search proficiency (low, high). This analysis indicated that the main effect of difficulty was significant, $F(1,20)=35.97$, $p<.001$, as were the main effects of strategy, $F(1,20)=$ $6.11, p<.02$, and proficiency, $F(1,20)=19.92, p<.001$. The two-way and three-way interactions all pointed to synergistic influences among these three factors: difficulty $\times$ strategy, $F(1,20)=6.47, p<.02$; difficulty $\times$ proficiency, $F(1,20)=3.13, p<.09$; strategy $\times$ proficiency, $F(1,20)=3.90, p<.06$; difficulty $\times$ strategy $\times$ proficiency, $F(1,20)=3.91, p<.06$. For example, the effect of strategy (i.e., active searchers were more efficient than passive searchers) was greater for hard than for easy search difficulties.

Analysis 2: Person perception ratings are sensitive to search proficiency, search strategy, and search difficulty, although to varying degrees The results show that person perception is indeed sensitive to these factors, resembling the main findings of the previous experiment at a broad brush. That is, indirect ratings of searcher activity were again more sensitive than direct ratings of searcher ability to individual differences between searchers. However, there were also important differences. Ratings of head movement frequency were more closely linked to these factors than were ratings of eye movement frequency. Ratings of positive emotion and interest showed an interaction effect involving strategy and proficiency, with searchers being judged as expressing the highest degree of positive emotion and interest when their search strategy matched their search proficiency (i.e., high-proficiency active searchers and lowproficiency passive searchers expressed the most positive emotion, whereas low-proficiency active searchers and high-proficiency passive searchers expressed the least). These conclusions were based on the following statistical analyses.

Interrater reliability The mean ratings of the video clips on each of the dimensions we tested generally yielded high interrater agreement (Cronbach's alpha for rated ability $=$ .880 , activity $=.823$, head $=.887$, eye $=.823$, emotion $=$ .935 , interest $=.877$ ). This strong agreement on all person perception rating scales indicates that raters were in consensus on these constructs of person perception.

Figure 8 shows the mean ratings for each of six rated dimensions as a function of search proficiency (low, high) and search strategy (active, passive). The search difficulty factor, although not shown here in order to 
Fig. 8 Mean ratings for each of six rated dimensions in Experiment 2 as a function of search proficiency (low, high) and search strategy (active, passive). Error bars are \pm 1 standard error of the mean
A. Global Behavior
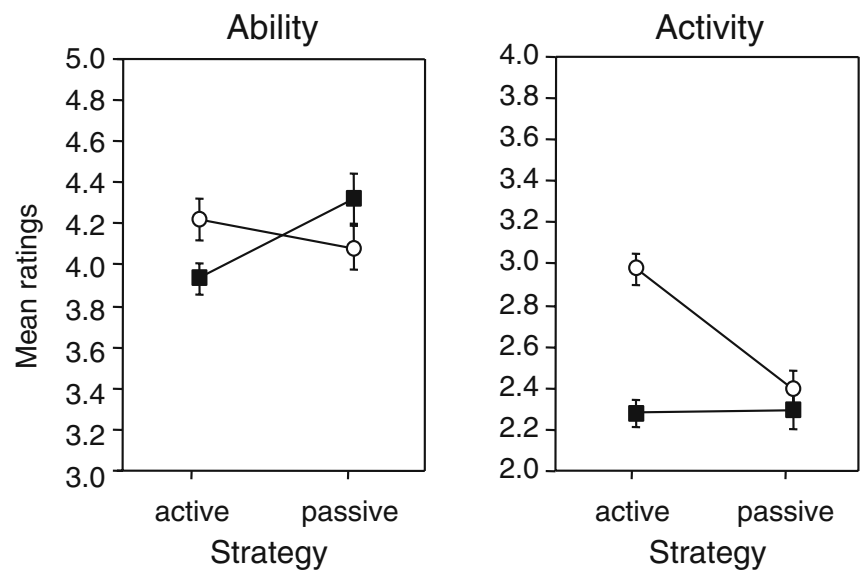

B. Local Behavior

Search Proficiency

○ High

- Low
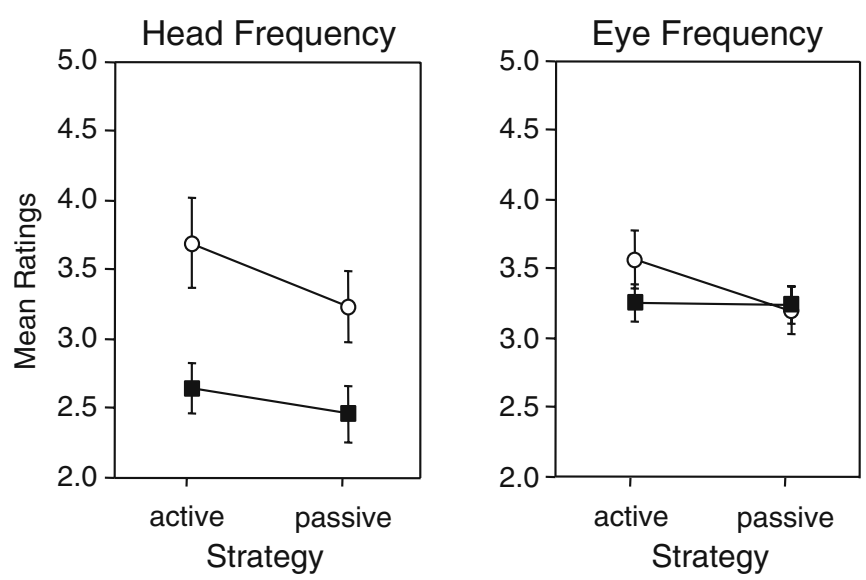

B. Mindset Attribution
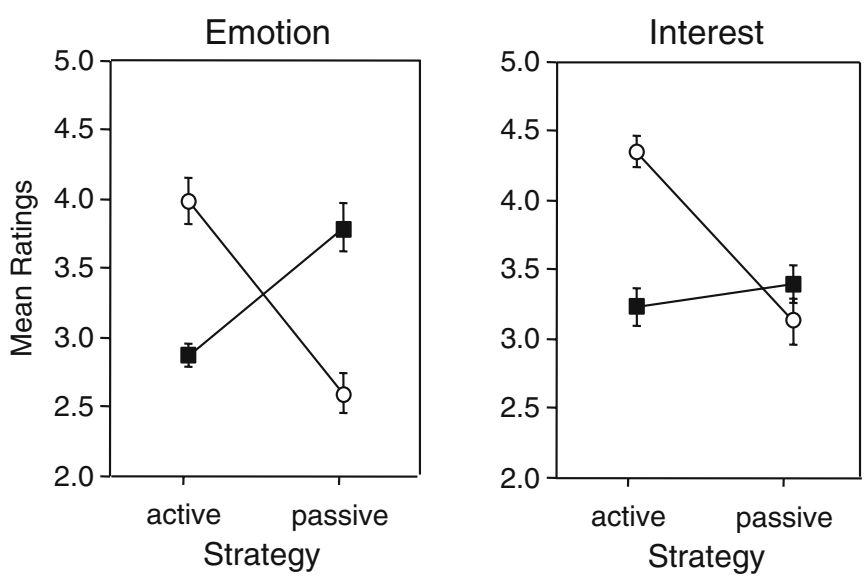

focus our analysis on individual differences in the ratings, also had an influence on many of the ratings, as we will describe below.

Ratings of global attribution The blind ratings of Phase 2 participants with regard to searcher activity were sensitive to both proficiency, $F(1,9)=38.79, p<.001$, and strategy, $F(1,9)=25.80, p<.001$, showing higher levels of rated activity for searchers of high proficiency and greater activity, but not to task difficulty, $p>.10$. Ratings of ability were not sensitive to either proficiency or strategy $(p>.10)$ but did show a significant sensitivity to difficulty, $F(1,10)=43.95, p<.01$, with higher levels of ability assigned to clips in which the targets were easier to find.

Ratings of local behavior Ratings of head movement frequency were sensitive to all three factors [proficiency, $F(1,6)=43.77, p<.001$; strategy, $F(1,6)=12.99, p<.01$; difficulty, $F(1,6)=21.86, p<.001$ ], with higher ratings assigned to higher proficiency searchers and active search- 
ers and in the harder search conditions. Ratings of eye movement frequency were sensitive only to the factor of strategy, $F(1,6)=15.92, p<.01$, with higher ratings assigned to searchers adopting an active strategy than to those adopting a passive strategy.

Mind-set attribution ratings Ratings of interest were sensitive to all three factors [proficiency, $F(1,9)=7.82, p<.02$; strategy, $F(1,9)=23.91, p<.01$; difficulty, $F(1,9)=$ $31.83, p<.01]$, with higher ratings assigned to higher proficiency searchers and active searchers and in the harder search conditions. Ratings of emotion upon detection of the target were sensitive to strategy [higher ratings in the active than in the passive strategy, $F(1,9)=5.87, p<.05]$, and to difficulty [higher ratings in the hard than in the easy condition, $F(1,9)=87.87, p<.01]$.

Table 5 shows the outcome of a head-to-head-comparison between the performance measure of search efficiency in Phase 1 and the six person perception ratings in Phase 2. These analyses were conducted as multiple regression analyses, where, for each measure, we examined the simple correlation coefficients for the orthogonal predictors of proficiency (high, low) and strategy (active, passive). The unit of prediction was the mean search efficiency for each of the 24 participants in the hard search conditions, where individual differences in the performance of the searchers were the greatest.

Table 5 Multiple regression analyses of individual differences in proficiency (based on a median split of search efficiency in each strategy group) and strategy (based on the random assignment of participants to either an active or a passive strategy condition) as predicted by search efficiency and by the six third-person ratings in Phase 2 of Experiment 2. The data include all 24 searchers and are taken from trials in the hard search condition, where individual differences were the greatest

\begin{tabular}{llll}
\hline Measure & Proficiency $r$ & Strategy $r$ & $R^{2}$ \\
\hline $\begin{array}{l}\text { Phase 1 Performance: } \\
\text { Search efficiency }\end{array}$ & $.597^{* * *}$ & $.410^{* * *}$ & $.525^{* * *}$ \\
& & & \\
Phase 2 Global Behavior Ratings & & \\
Ability & .239 & .239 & .114 \\
Activity & $.392^{* *}$ & $.336^{*}$ & $.267^{* *}$ \\
& & & \\
Phase 2 Local Behavior Ratings & & $.349^{* *}$ \\
Head movement & $.558^{* *}$ & .193 & .025 \\
Eye movement & .100 & .123 & \\
& & & .058 \\
Phase 2 Mindset Ratings & .241 & .014 & \\
Emotion & .138 & & \\
Interest & &
\end{tabular}

The last column in Table 5 indicates the extent to which variance among individual searchers is explained by the factors of proficiency and strategy. It shows that the performance measure (i.e., search efficiency) accounted for $52.5 \%$ of the individual differences in search performance and that the efficiency measure was significantly sensitive to individual differences arising from both proficiency, $r=.597, t(21)=3.97, p<.001$, and strategy, $r=.410, t(21)=2.73, p<.01$.

By way of comparison, among the global attribution ratings, activity was the most sensitive rating scale, accounting for $26.7 \%$ of the variance and also showing independent sensitivity to proficiency, $r=.392, t(21)=$ 2.10, $p<.05$, and marginally to strategy, $r=.336, t(21)=$ $1.80, p<.09$. Ability ratings were much less sensitive, accounting for only $11.4 \%$ of the variance and showing no significant sensitivity to either proficiency or strategy $(p>.25)$.

Among the local behavioral ratings, frequency of head movements (34.9\% of variance explained) showed greater sensitivity to individual differences in performance than did frequency of eye movements ( $2.5 \%$ of variance explained).

This contrasts with the finding in Experiment 1, where rated eye movement frequency accounted for more of the individual differences in search performance than did rated head movement frequency. This finding therefore points to a shift in the relative importance of eye and head activity, depending on the context in which search takes place.

Finally, the two ratings of mind-set showed very little sensitivity to search performance on their own $(5.8 \%$ and $9.7 \%$ of variance explained, respectively), but each of them was involved in a significant $(p<.01)$ two-way interaction involving proficiency and strategy factors. When these interaction terms were included in the regression analysis, the explained variance increased to $35.7 \%$ (emotion) and $24.7 \%$ (interest). These interactions are shown in Fig. 8 for the emotion and interest ratings. This pattern in the ratings indicates that searchers were judged as expressing the highest degree of positive emotion (and interest) when the cognitive strategy they adopted matched their search proficiency. That is, high-proficiency active searchers and low-proficiency passive searchers expressed the most positive emotion, whereas low-proficiency active searchers and high-proficiency passive searchers expressed the least.

Such a congruency effect on emotional expression is reminiscent of fluency theory (Reber et al., 1998), which proposes that more fluent processing produces more positive responses. In this framework, searchers experienced maximum enjoyment when their proficiency and strategy were aligned, and importantly, this increased enjoyment was visible in searchers' overt behaviors and expressions. The present results suggest that emotion-a "warm" factor that has not previously been considered in 
studies of visual search — has an important influence on cognitive performance in a natural-setting visual search for everyday objects.

Analysis 3: The person perception rating that best predicts natural setting search performance is either activity or head movement frequency In these analyses, we asked which person perception ratings, either alone or in combination, did the best job of accounting for variance in the detailed search performance data of Phase 1. Because some of these rating scales were also correlated strongly with one another, we used a simultaneous regression procedure to help determine which rating scales contained the greatest amount of unique information with regard to search performance. This analysis indicated that $32 \%$ of all the variance in the performance of individual searchers could be accounted for by activity ratings and that $24 \%$ could be accounted for by head movement ratings.

Table 6 shows a table of cross-correlations for the six rating scales tested in this experiment. Once again, there was much overlap in these rating scales, with, for example, activity ratings and head movement frequency ratings having much in common $(r=.752)$ and emotion and interest sharing a great deal of variance $(r=.663)$. In contrast, eye movement and head movement frequency are relatively uncorrelated $(r=.208)$.

Table 7 shows the results of our regression analyses. The first column shows the simple correlation between mean individual search efficiency scores and the six person perception ratings. To see which of these ratings contributed uniquely to the individual differences in search efficiency, we entered all of them as predictors in a multiple regression model in which the efficiency scores of the 24 searchers represented the outcome variable. The full model involving all six ratings yielded an $R^{2}$ value of $.362, F(6$, $17)=1.61, p>.20$. By systematically removing ratings that contributed least to the total variance explained, as indicated by their partial coefficients, we found that two reduced models, each involving only one of the ratings, accounted for a similar amount of variance. These are shown in the last two columns of Table 7. The model including only the activity ratings yielded an $R^{2}$ value of $.320, F(1,22)=10.35, p<.01$, and the model including only the head movement ratings yielded an $R^{2}$ value of $.243, F(1,22)=7.07, p<.02$. Each of these reduced models did not differ significantly from the full model, and they were also highly correlated with one another $(r=$ .752 ), indicating that ratings of either activity or of head movement frequency could be used to predict search efficiency in this experiment.

\section{General discussion}

This study demonstrates the merits of using person perception to understand important aspects of visual search. This was accomplished in several steps, as illustrated in Fig. 1. After first establishing that three different factors had an influence on search performance (analysis 1), we demonstrated that some person perception ratings of thirdperson observers were sensitive to these same factors (analysis 2). For example, in Experiment 1, where search efficiency scores accounted for $68 \%$ of the individual differences in search performance, blind ratings of searcher activity accounted for $49 \%$ of the same variance on their own, ratings of eye movement frequency accounted for $30 \%$, and ratings of emotion accounted for $33 \%$. Moreover, when the unique contributions of each person perception rating scale was compared with one another (analysis 3 ), over $60 \%$ of all the variance in the performance of individual searchers could be accounted for by just two rating scales: the frequency of eye movements and the expression of positive emotion in the faces of searchers.

In Experiment 2, where search was conducted in a natural everyday setting with far fewer trials performed by each searcher, we still observed significant sensitivity for the individual differences in search in both the performance measures (analysis 1) and the person perception measures (analysis 2). Whereas search efficiency in this experiment accounted for $52 \%$ of the variance, ratings of searcher activity were able to account for $27 \%$, and ratings of head movement frequency were able to account for $35 \%$. When the unique contributions of each rating scale were compared in predicting individual differences in mean search efficiency (analysis 3), the results showed that over 32\% of
Table 6 Correlations among the six third-person rating scales in Phase 2 of Experiment 2

\begin{tabular}{|c|c|c|c|c|c|c|}
\hline & Ability & Activity & Head & Eyes & Emotion & Interest \\
\hline Ability & & .095 & .006 & .200 & .007 & .103 \\
\hline Activity & & & $.752 * *$ & .326 & .074 & $.483 *$ \\
\hline Head & & & & .208 & .279 & .239 \\
\hline Eyes & & & & & .115 & .191 \\
\hline Emotion & & & & & & $.663 * *$ \\
\hline Interest & & & & & & \\
\hline
\end{tabular}

$* p<.05$

$* * p<.01$

$* p<.05$
$* * p<.01$


Table 7 Multiple regression models using person perception ratings to predict individual differences in search efficiency in Experiment 2

\begin{tabular}{|c|c|c|c|c|}
\hline \multirow[t]{2}{*}{ Ratings } & \multirow[t]{2}{*}{ Simple $r$} & \multirow{2}{*}{$\begin{array}{l}\text { Full model } \\
\text { Partial } r\end{array}$} & \multirow{2}{*}{$\frac{\begin{array}{l}\text { Reduced } \\
\text { model }\end{array}}{\text { Simple } r}$} & \multirow{2}{*}{ 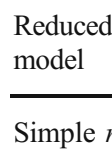 } \\
\hline & & & & \\
\hline Ability & .085 & .049 & & \\
\hline Activity & $.566 * * *$ & .280 & $.566 * * *$ & \\
\hline Head & $.493 * *$ & .064 & & $.493 * *$ \\
\hline Eyes & .268 & .073 & & \\
\hline Emotion & .217 & .143 & & \\
\hline Interest & .180 & .049 & & \\
\hline$R^{2}$ & & .362 & .320 & .243 \\
\hline
\end{tabular}

The first column shows simple correlations between mean individual search efficiency scores and the six ratings. Asterisks refer to statistical significance of these correlations: ${ }^{*} p<.10,{ }^{* *} p<.05$, $* * * p<.01$. Partial $r$ is a relative measure of unique variation for each predictor; $R^{2}$ is the proportion of variance in the individual search efficiency that is accounted for by the predictors in each model

all the variance in the performance of individual searchers could be accounted for by using just the activity rating scale on its own, which was strongly correlated with the head movement frequency scale.

The role of activity in search The results of both experiments pointed to the importance of visible motor activity in successful search; specifically, more frequent eye movements were positively correlated with search efficiency on a computer screen (Experiment 1), and more frequent head movements were correlated with search efficiency in a real office (Experiment 2). We also found that in both experiments, participants who were instructed to be active searchers made more eye and head movements than did participants who were instructed to be passive searchers.

It is worth noting that there was no definitive basis for predicting whether head or eye movements would be more important for search. For example, in both experiments, some amount of eye movement was almost certainly required, simply because of the limits of visual acuity, relative to the size of the displays. That is, target objects that were about $1^{\circ}$ in size needed to fall within the $2^{\circ}-3^{\circ}$ foveal region of the eye in order to be identified. Indeed, the literature is not especially informative as to whether search success is generally linked more strongly to head or eye movements, or even as to the more general question of whether more or fewer movements of any kind are of greater benefit. Two reasons for this are that very few studies of search have been conducted on screens that encompass a large region of the visual field, and much of the research has been conducted with participants whose head movements were artificially restricted by use of a chinrest.
Moreover, on the question of relative movement frequency and search success, the previous literature is decidedly mixed. Some of the existing research indicates that efficient search (rapid and accurate target identification) is associated with fewer overall eye movements during search (Boot et al., 2009; Schoonard, Gould, \& Miller, 1973; Shapiro \& Raymond, 1989; Togami, 1984; Watson et al., 2010). Consistent with this idea, some reports even indicate that preventing searchers from making any eye movements at all can sometimes be beneficial (Klein \& Farrell, 1989; Zelinsky \& Sheinberg, 1997). Yet, at the same time, other reports indicate that more frequent eye movements are advantageous in search, especially when targets viewed in peripheral vision are difficult to distinguish from distractors (Boot et al., 2009).

Previous research on the role of search strategies also does not clarify the relationship between activity and search success. There are reports of a passive search advantage, where a passive strategy is associated with more efficient visual search (Smilek et al., 2006b). A recent study has even established that participants instructed with a passive strategy wait longer before beginning to move their eyes in a search task and then make fewer saccades overall than actively instructed participants (Watson et al., 2010). The present finding of an active search advantage (i.e., participants instructed to search actively were more efficient that participants instructed to search passively) therefore stands in sharp contrast to this previous research.

We interpret the finding of an active search advantage in the present study as indicating that successful search on large displays depends on how actively the searcher reorients his or her gaze from moment to moment while searching for an object. This may have been overlooked in previous studies simply because only small displays were tested and/or because target identification did not depend critically on gaze reorientation. This means that although active gaze reorientation may be detrimental to search under those special circumstances, it may generally be beneficial for larger and more complex displays, such as those tested here.

The role of emotion in search The person perception measures in this study allowed us to investigate how positive emotions are linked to success in visual search, a task that had previously been treated as though it was devoid of emotion. We found that positive emotions play an important role in visual search: The computer search in Experiment 1 indicated a positive correlation between positive emotional expressions and successful search, and the office search in Experiment 2 pointed to a congruency effect involving the emotional experience and performance of searchers. Reconciling these differences will likely come about only through additional research, but here we offer a tentative hypothesis to help guide future work. 
One way to understand the apparent difference in the two patterns of results is to consider the possibility that both effects exist in principle but that there was not sufficient power to detect both of them in each of the present experiments. Under this hypothesis, positive emotions are always linked to more efficient cognitive processing, and equally important, proficiency-strategy congruency always plays a role in the emotional experience of a study participant. Positive emotions and fluent cognition are simply different directions on the same two-way street, such that positive emotions generally benefit cognitive processing and fluent cognition generally alters one's emotional experience in a positive way. From this perspective, the way the results for emotion in Experiments 1 and 2 differ from one another is more a matter of emphasis than of kind. The data in Experiment 1 seem to show the emotion-to-cognition connection most strongly, and those in Experiment 2 reveal the link from cognitive performance to emotional experience.

If this view is correct, future studies altering the emotional experience of a study participant in advance of a task should have a direct effect on his or her performance. Conversely, altering the performance efficiency of a study participant (e. g., through increasing expertise or external manipulations of ease of processing) should have a direct and positive effect on his or her emotional experience. Both of these possibilities have already been suggested in previous research and theory, with flow theory (Csikszentmihalyi \& Rathunde, 1993) focusing more on the influences of emotion on cognition and action and fluency theory (Reber et al., 1998) focusing more on the influence of cognition on emotion. What has not been done so far is to systematically examine these two directions of influence in standard tasks of cognition.

It is also possible that a difference in the social contexts of our two experiments contributed to the divergent pattern of findings for emotion (Aiello \& Svec, 1993; Bond \& Titus, 1983; Zajonc, 1965). Experiment 1 took place in social isolation, once the instructions had been given, whereas Experiment 2 involved the continued presence of a live experimenter who interacted with the participant between trials. This difference may have played a role, but any strong interpretation must be treated with caution, because there were also numerous other differences between the experiments.

The merits of person perception for studying visual cognition There were two primary findings of this study with regard to using person perception in the study of cognition: (1) Person perception ratings are differentially sensitive to the experimental factors of interest (i.e., some predicted search performance better than did others), and (2) person perception is an inexpensive, effective, and unobtrusive way to measure cognition and emotion.
The results of both experiments showed that indirect ratings of search behavior, including ratings of global activity and more detailed ratings of eye and head movements, were all more strongly predictive of search performance than was a direct rating of a searcher's ability. Although this might seem to be a weakness of person perception, in that not all ratings were sensitive to the factors influencing search performance, we view it as revealing the strength in the approach. Indeed, if all person perception ratings were equally sensitive to all factors influencing search performance, it would mean that there are no differentiable constructs of efficient visual search. Instead, the finding that rating scales are differentially sensitive points to the possibility of teasing apart the contributing factors to search, using third-person measures.

Consider, for example, that ability ratings correlate with a different set of person perception measures than do the eye and head movement frequency ratings. In Experiment 1 , ability ratings were most strongly correlated with ratings of interest $(r=.694)$. However, bear in mind that ratings of eye movement frequency and positive emotion were actually the superior predictors of search performance in this experiment. Taken together, these results suggest that the ability ratings tap into different dimensions of person perception than those actually contributing to search performance. An interesting question for future research may well be, what visible features were raters using to make judgments of ability? Perhaps participants were judging personal attractiveness, following the common misconception that "what is beautiful is good" (Dion, Berscheid, \& Hatfield, 1972).

The second strength of person perception measures lies in the demonstration that humans can serve as excellent eye and head trackers. The value of this to research becomes glaringly apparent when one considers the limitations of modern eye and head trackers for research in natural settings. First, there is the cost factor: All conventional eye trackers are expensive. Desktop-mounted eye trackers assume that participants are seated at a computer, that they are not moving their heads, and that they know that their eyes are being tracked. The mobile eye trackers currently available cannot dissociate between the head and eye movements that contribute to gaze location in the scene. There are dedicated head trackers available, but these are not routinely or easily integrated with eye trackers, and they are of course also costly.

By way of comparison, person perception ratings of eye and head movements are cheap; they do not limit participant mobility; and they can be applied without specific awareness that eye and head movements are the focus of study. Moreover, they can be as accurate as automated eye trackers under some conditions (Anderson, Risko, \& Kingstone, 2011), and they may avoid the potential pitfalls of eye 
tracker awareness (Risko \& Kingstone, 2011). Moreover, the present data show that person perception ratings dissociate the relative contributions of eye and head activity, something that is not easily accomplished with conventional eye- and head-tracking equipment.

In conclusion, we present these findings as a proof-ofconcept that person perception, which has a wellestablished tradition of study within social psychology (Rule et al., 2009; Weisbuch \& Ambady, 2011), can be harnessed to assist in the study of basic cognitive processes. We believe that this finding, and our interpretation of it, opens up a potentially rich new world of measurement for cognitive research. The challenge for the future will be to determine the reliability and range of third-person observational skills in leading to a better understanding of firstperson cognition.

Acknowledgements We thank three anonymous reviewers for their constructive comments on an earlier version of the manuscript. This research was supported with grants from the Natural Sciences and Engineering Research Council (Canada), a Discovery Research Grant to J. Enns, and an Alexander Graham Bell Canada Graduate Scholarship (CGS) to A. Brennan. The authors thank Nancy Deng, Maggie Dong, KL Ta, and Maya Whitehead for enthusiastic data collection and conscientious video editing.

\section{Appendix}

Rating scales used in Phase 2 of Experiments 1 and 2

\section{Ratings of global attribution}

Energy level:

Please rate the ENERGY LEVEL of participants as they search the target: ( 1 = VERY LOW ENERGY; $6=$ VERY HIGH ENERGY)

Search Ability:

Some participants were POOR searchers - they were SLOW and INACCURATE. Some participants were GOOD searchers - they were FAST and ACCURATE.

Please rate the ABILITY of participants as they search the target: ( 1 = POOR SEARCHER; 6 = GOOD SEARCHER)

Search Activity:

Before beginning the experiment, each participant was instructed to search for the hidden object either ACTIVELY or PASSIVELY.

The ACTIVE instructions were as follows:

"The best strategy for this task, and the one that we want you to use in this study, is to be as active as possible and to "search" for the target as you look [at the screen in Experiment 1/around the room in Experiment 2]. The idea is to deliberately direct your attention to find the target. Sometimes people find it difficult or strange to direct their attention - but we would like you to try your best. Try to respond as quickly and accurately as you can while using this strategy. Remember, it is very critical for this experiment that you actively search for the target."

The PASSIVE instructions were as follows:

"The best strategy for this task, and the one that we want you to use in this study, is to be as receptive as possible and let the target "pop" into your mind as you look [at the screen in Experiment 1/around the room in Experiment 2]. The idea is to let your intuition determine how you find the target. Sometimes people find it difficult or strange to tune into their "gut feelings" but we would like you to try your best. Try to respond as quickly and accurately as you can while using this strategy. Remember, it is very critical for this experiment that you let the target just "pop" into your mind."

Please indicate which instructions you believe the participant received: $(1=$ confident passive; $2=$ guess passive; 3 = guess active; $4=$ confident active)

\section{Ratings of local behavior}

Head Movement:

Please rate the amount of HEAD MOVEMENT made by participants as they searched for target: $(1=$ NO HEAD MOVEMENT; 6 = MUCH HEAD MOVEMENT)

Eye Movement:

Please rate the amount of EYE MOVEMENT made by participants as they searched for target: $(1=\mathrm{NO}$ EYE MOVEMENTS; 6 = MANY EYE MOVEMENTS)

\section{Ratings of mind-set}

Interest:

Please rate how INTERESTED participants appeared as they searched for the target: $(1=$ BORED; $6=$ INTERESTED $)$

Positive Emotion:

Please rate the amount of PLEASURE AND SATISFACTION shown by participants upon finding the target: (1 = UNHAPPY/DISSATISFIED; $6=$ VERY HAPPY/ SATISFIED)

\section{References}

Aiello, J. R., \& Svec, C. M. (1993). Computer monitoring and work performance: Extending the social facilitation framework to electronic presence. Journal of Applied Social Psychology, 23, $537-548$.

Ambady, N., Hallahan, M., \& Rosenthal, R. (1995). On judging and being judged accurately in zero acquaintance situations. Journal of Personality and Social Psychology, 69, 518-529.

Ambady, N., Bernieri, F. J., \& Richeson, J. A. (2000). Toward a histology of social behavior: Judgmental accuracy from thin slices of the behavioral stream. In M. P. Zanna (Ed.), 
Advances in experimental social psychology (Vol. 32, pp. 201-271). San Diego: Academic.

Anderson, N.C., Risko, E., \& Kingstone, A. (2011). Exploiting human gaze sensitivity for tracking the eyes. Behavior Research Methods.

Ashby, F. G., Isen, A. M., \& Turken, U. (1999). A neuropsychological theory of positive affect and its influence on cognition. Psychological Review, 106, 529-550.

Beck, M. R., Levin, D. T., \& Angelone, B. L. (2007). Metacognitive errors in change detection: Lab and life converge. Consciousness and Cognition, 16, 58-62.

Bond, C. F., \& Titus, L. J. (1983). Social facilitation: A meta-analysis of 241 studies. Psychological Bulletin, 94, 265-292.

Boot, W. R., Becic, E., \& Kramer, A. F. (2009). Stable individual differences in search strategy?: The effect of task demands and motivational factors on scanning strategy in visual search. Journal of Vision, 9(3, Art. 7), 1-16.

Borkenau, P., \& Liebler, A. (1995). Observable attributes as manifestations and cues of personality and intelligence. Journal of Personality, 63, 1-25.

Borkenau, P., Mauer, N., Riemann, R., Spinath, F. M., \& Angleitner, A. (2004). Thin slices of behavior as cues of personality and intelligence. Journal of Personality and Social Psychology, 86, 599-614.

Carney, D. R., Colvin, C. R., \& Hall, J. A. (2007). A thin slice perspective on the accuracy of first impressions. Journal of Research in Personality, 41, 1054-1072.

Csikszentmihalyi, M., \& Rathunde, K. (1993). The measurement of flow in everyday life: Toward a theory of emergent motivation. In J. E. Jacobs (Ed.), Nebraska Symposium on Motivation 1992 (Vol. 40, pp. 57-97). Lincoln: University of Nebraska Press

Derryberry, D., \& Reed, M. A. (1998). Anxiety and attentional focusing: Trait, state and hemispheric influences. Personality and Individual Differences, 25, 745-761.

Derryberry, D., \& Tucker, D. M. (1994). Motivating the focus of attention. In P. M. Neidenthal \& S. Kitayama (Eds.), The heart's eye: Emotional influences in perception and attention (pp. 167196). San Diego: Academic.

Dijksterhuis, A. (2004). Think different: The merits of unconscious thought in preference development and decision making. Journal of Personality and Social Psychology, 87, $586-598$

Dion, K., Berscheid, E., \& Hatfield, E. (1972). What is beautiful is good. Journal of Personality and Social Psychology, 24, 285-290.

Fredrickson, B. L. (2003). The value of positive emotions. American Scientist, 91, 330-335.

Gasper, K., \& Clore, G. L. (2002). Attending to the big picture: Mood and global versus local processing of visual information. Psychological Science, 13, 34-40.

Gladwell, M. (2007). Blink: The power of thinking without thinking. New York: Little, Brown.

Gray, J. R. (2001). Emotional modulation of cognitive control: Approach-withdrawal states double-dissociate spatial from verbal two-back task performance. Journal of Experimental Psychology: General, 130, 436-452.

Gray, J. R. (2004). Integration of emotion and cognitive control. Current Directions in Psychological Science, 13, 46-48.

Greenwald, A. G., Poehlman, T. A., Uhlmann, E. L., \& Banaji, M. R. (2009). Understanding and using the Implicit Association Test: III. Meta-analysis of predictive validity. Journal of Personality and Social Psychology, 97, 17-41.

Henderson, J. M. (Ed.). (2005). Real-world scene perception. New York: Psychology.

Hutchins, E. (1995). Cognition in the wild. Cambridge: MIT.

Jefferies, L. N., Smilek, D., Eich, E., \& Enns, J. T. (2008). Emotional valence and arousal interact in the control of attention. Psychological Science, 19, 290-295.
Kane, M. J., Brown, L. H., Little, J. C., Silvia, P. J., MyinGermeys, I., \& Kwapil, T. R. (2007). For whom the mind wanders, and when: An experience-sampling study of working memory and executive control in daily life. Psychological Science, 18, 614-621.

Kingstone, A., Smilek, D., \& Eastwood, J. D. (2008). Cognitive ethology: A new approach for studying human cognition. British Journal of Psychology, 99, 317-345.

Klein, R., \& Farrell, M. (1989). Search performance without eye movements. Perception \& Psychophysics, 46, 476-482.

Levin, D. T., \& Beck, M. R. (2004). Thinking about seeing: Spanning the difference between metacognitive failure and success. In D. T. Levin (Ed.), Thinking and seeing: Visual metacognition in adults and children (pp. 121-143). Cambridge: MIT.

Maison, D., Greenwald, A. G., \& Bruin, R. H. (2004). Predictive validity of the Implicit Association Test instudies of brands, consumer attitudes, and behavior. Journal of Consumer Psychology, 14, 405-415.

Naumann, L. P., Vazire, S., Rentfrow, P. J., \& Gosling, S. D. (2009). Personality judgments based on physical appearance. Personality and Social Psychology Bulletin, 35, 1661-1671.

Nummenmaa, L., \& Calder, A. J. (2009). Neural mechanisms of social attention. Trends in Cognitive Sciences, 13, 135-143.

Orne, M. T. (1962). On the social psychology of the psychological experiment: With particular reference to demand characteristics and their implications. American Psychologist, 17, 776-783.

Palermo, R., \& Rhodes, G. (2007). The perception of emotion and social cues in faces. Neuropsychologia, 45, 75-92.

Phillips, L. H., Bull, R., Adams, E., \& Fraser, L. (2002). Positive mood and executive function: Evidence from Stroop and fluency tasks. Emotion, 2, 12-22.

Reber, R., Winkielman, P., \& Schwarz, N. (1998). Effects of perceptual fluency on affective judgments. Psychological Science, 9, 45-48.

Risko, E., \& Kingstone, A. (2011). Eyes wide shut: Implied social presence, eye tracking and attention. Attention, Perception, \& Psychophysics, 73, 291-296.

Rule, N., Macrae, C. N., \& Ambady, N. (2009). Ambiguous group membership is extracted automatically from faces. Psychological Science, 20, 441-443.

Schoonard, J. W., Gould, J. D., \& Miller, L. A. (1973). Studies of visual inspection. Ergonomics, 16, 365-379.

Shapiro, K. L., \& Raymond, J. E. (1989). Training of efficient oculomotor strategies enhances skill acquisition. Acta Psychologica, 71, 217-242.

Silvia, P. J. (2008). Interest, the curious emotion. Current Directions in Psychological Science, 17, 57-60.

Smilek, D., Dixon, M. J., \& Merikle, P. M. (2006a). Revisiting the category effect: The influence of meaning and search strategy on the efficiency of visual search. Brain Research, 1080, 73-90.

Smilek, D., Enns, J. T., Eastwood, J. D., \& Merikle, P. A. (2006b). Relax! Cognitive style influences visual search. Visual Cognition, 14, 543-564.

Smilek, D., Weiheimer, L., Kwan, D., Reynolds, M., \& Kingstone, A. (2009). Hiding and finding: The relationship between visual concealment and visual search. Attention, Perception, \& Psychophysics, 71, 1793-1806.

Togami, H. (1984). Affects on visual search performance of individual differences in fixation time and number of fixations. Ergonomics, 27, 789-799.

Townsend, J. T., \& Ashby, F. G. (1983). Stochastic modeling of elementary psychological processes. New York: Cambridge University Press.

Tunnell, G. B. (1977). Three dimensions of naturalness: An expanded definition of field research. Psychological Bulletin, 84, 426- 437. 
Wadlinger, H. A., \& Isaacowitz, D. M. (2006). Positive mood broadens visual attention to positive stimuli. Motivation and Emotion, 30, 87-99.

Watson, M. R., Brennan, A. A., Kingstone, A., \& Enns, J. T. (2010). Looking versus seeing: Strategies alter eye movements during visual search. Psychonomic Bulletin \& Review, 17, 543-549.

Weisbuch, M., \& Ambady, N. (2011). Thin slice vision. In R. B. Adams, Jr., N. Ambady, K. Nakayama, \& S. Shimojo (Eds.), The science of social vision. New York: Oxford University Press.

Wolfe, J. M. (1998). What can 1,000,000 trials tell us about visual search? Psychological Science, 9, 33-39.
Wolfe, J. M., Horowitz, T. S., Van Wert, M. J., Kenner, N. M., \& Kibbi, M. (2007). Low target prevalence is a stubborn source of errors in visual search tasks. Journal of Experimental Psychology: General, 136, 623-638.

Wolfe, J. M., \& Van Wert, M. (2010). Varying target prevalence reveals two dissociable decision criteria in visual search. Current Biology, 20, 121-124.

Zajonc, R. B. (1965). Social facilitation. Science, 149, 269-274.

Zelinsky, G. J., \& Sheinberg, D. L. (1997). Eye movements during parallel-serial visual search. Journal of Experimental Psychology: Human Perception and Performance, 23, 244-262. 